\title{
On the Synchronization of Two Eccentric Rotors with Common Rotational Axis: Theory and Experiment
}

\author{
Xiaozhe Chen, Xiangxi Kong, Xueliang Zhang, Lingxuan Li, and Bangchun Wen
}

School of Mechanical Engineering and Automation, Northeastern University, Shenyang 110819, China

Correspondence should be addressed to Xiaozhe Chen; xzchen1986@163.com

Received 28 March 2016; Revised 22 May 2016; Accepted 22 June 2016

Academic Editor: Hassan Haddadpour

Copyright ( 2016 Xiaozhe Chen et al. This is an open access article distributed under the Creative Commons Attribution License, which permits unrestricted use, distribution, and reproduction in any medium, provided the original work is properly cited.

\begin{abstract}
We study synchronization of two eccentric rotors (ERs) with common rotational axis in the vibration system of the far-resonant spatial motion. We deduce the dimensionless coupling equation of two ERs with applying the average method of small parameters. We convert the synchronization problem into the existence and stability of solving the zero solutions for the dimensionless coupling equations. By introducing the synchronization torque and the difference between the residual torques of two motors, we obtain the synchronization condition that two ERs achieve the synchronized motion. We derive the stability condition of the synchronized motion, which satisfies Routh-Hurwitz criterion. We discuss numerically the choosing motion feature of the vibration system, which indicates that the vibration system has two steady motion modes. The synchronization torque forces the phase difference to approach $\pi$ when the structural parameters of the vibration system satisfy the condition of the spatial cone motion, and the synchronization torque drives the phase difference to approach zero when those satisfy the condition of the spatial circle motion. Finally, through the comparison and analysis of experimental data, the structural parameters of the vibration system satisfying the above two conditions can guarantee the synchronization stability for two ERs.
\end{abstract}

\section{Introduction}

In vibration utilization engineering, synchronization of ERs (also called exciter) is a special phenomenon of the nonlinear vibration system, which is used to design the vibration conveyer, the vibration screen, the vibration centrifuge, and so forth [1]. The vibration mill is a kind of grinding equipment with high efficiency and energy saving, which has been widely used in many fields, such as metallurgy, chemical, mining, medical, ceramics, cement. In order to satisfy the increasing of powder processing, the vibration mill is large-scale development. However, for the large-scale vibration mill, its longer barrel is not driven by only one motor. There are many questions about the design of this vibration mill excited by multiple ERs, for example, two motors drive separately two ERs running in synchronization state to replace forced synchronization. Hence, applying the synchronization theory to design the large-scale vibration mill can simplify structure of the vibration mill, decrease production cost of that, and enhance work efficiency of that.
For a long time, utilizing the advantage of synchronization in engineering and technology fields has been the main aims of scholars, who have paid attention to synchronization of two or more ERs. When it comes to synchronization, we may date back to that of pendulum clocks found firstly by Huygens [2]. In the 1960s, Blekhman first clarified synchronization principle of two ERs in a vibration system and has successfully solved some problem about synchronization [3-8]. Since then, there are many excellent results which pushed the researches of the synchronization theory. Wen et al. selected the phase difference of ERs as object and applied the method of integral average to simplify the solution of synchronization [1]. Based on this concept, Zhao et al. applied the average method of small parameters to obtain the synchronization condition and the synchronization stability for two ERs [9-11]. This method considers the effect of damping and dynamic characteristics of induced motor. By applying the average method of small parameters, Zhang deduced the condition that vibration synchronization transmission for two ERs with reverse rotational direction and gave the result of the 


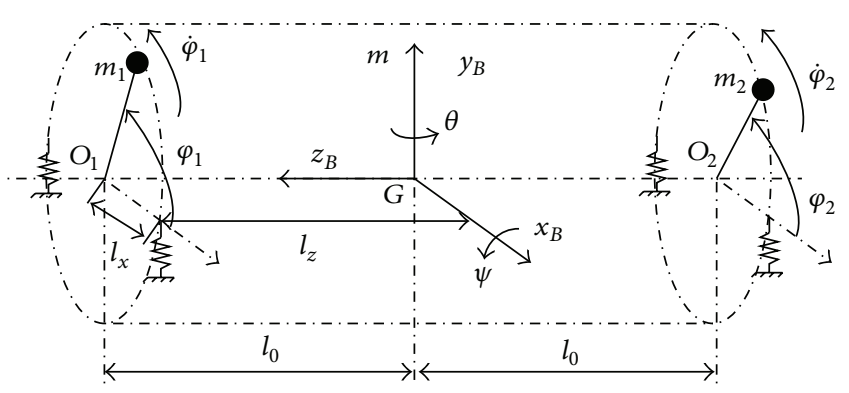

(a)

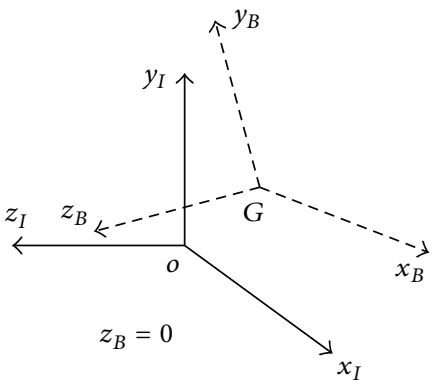

(b)

Figure 1: Dynamical model of the vibration system: (a) schematic diagram of structure; (b) the coordinate frames: $E_{I}=\left\{\begin{array}{lll}O & x_{I} \quad y_{I} & z_{I}\end{array}\right\}$, the earth fixed inertial frame, $E_{B}=\left\{\begin{array}{llll}O & x_{B} & y_{B} & z_{B}\end{array}\right\}$, the body fixed frame.

select motion characteristic for the vibration system driven by two ERs with same rotational direction $[12,13]$. He also gave the result that using three ERs instead of two on a single base cannot improve the effective power in the far-resonant vibration system [14]. There are also scholars who applied numerical analysis to study the synchronization problems. Balthazar analyzed the nonlinear phenomenon of synchronization of two nonideal sources by means of numerical simulations $[15,16]$. Bonkobara et al. applied shooting method to solve the displacement response curves of the vibration system to judge whether the vibration system achieves synchronization [17]. Rumyantsev and Tarasov addressed some comments on the nonlinear dynamics of vibration transport machines by the numerical simulation [18].

The above proposed result all selected two ERs or multiple ERs in a vibration system of plane motion as the object. However, there are few researches for spatial motion. Zhao et al. investigated the coupling dynamic characteristic for a vertical conveyer [19]. Miklós presented a dual-rotors exciter to produce vibrations by independently adjusting frequency and amplitude [20-22].

Hence, the research of synchronization of two ERs rotating with the common rotational axis is of great significance.

In this paper, synchronization of two ERs with the common rotational axis in the vibrating system of the farresonant spatial motion is investigated with applying the average method of small parameters. We addressed that this type of vibration system has the choosing motion feature, and the theory approach is verified to be useful and feasible by experiments. This paper is summarized as follows: first, the motion equations of the vibrating system are derived. Second, the synchronization condition and the synchronization stability are deduced. Third, the quantitative numeric discussions are provided. Fourth, experiments are shown. Finally, conclusions are given.

\section{Dynamic Model and Motion Equation of the Vibration System}

As shown in Figure 1(a), the dynamic model of the vibration system consists of a rigid body and two ERs. Four symmetrical isolation springs support the rigid body. Two induction motors drive two ERs with the same rotational direction, respectively, and their rotational axes of two ERs are common. $o_{1}$ and $o_{2}$ are the rotational centers of ER 1 and ER 2, respectively.

In Figure 1(b), let $E_{I}=\left\{\begin{array}{llll}O & x_{I} & y_{I} & z_{I}\end{array}\right\}$ denote the earth fixed inertial frame and let $E_{B}=\left\{\begin{array}{llll}G & x_{B} & y_{B} & z_{B}\end{array}\right\}$ denote the body fixed frame rigidly attached to the vibration system with origin $G$ in the center of mass.

Generally, because of the elastic foundation, the rigid body has six degrees of freedom. In engineering, the motion of this type of machine is designed for the spatial circle motion. According to the design, rubber spring is chosen. The spring of ROSTA only has the plane motion because of the bigger rigidity in the axis direction. In order to simplify analysis, the $z$-direction motion is considered to be fixed. Due to the symmetrical structure of the rigid body, the $z_{B}$-axis is assumed to coincide with the principle axis of the rigid body. Since points $G, o_{1}$, and $o_{2}$ are on $z_{B}$-axis, so the resultant force of the exciting forces passes the center of mass, and thus the rotation around $z_{I}$-axis is not existing.

To sum up, the vibration system has six degrees of freedom as follows: the rotational directions of ER 1 and ER 2 are denoted, respectively, by $\varphi_{1}$ and $\varphi_{2}$. The center of mass translations is $x$ and $y$, and two angular rotations are $\psi$ and $\theta$.

The coordinates of ER 1 and ER 2 are $\mathbf{x}_{1}^{\prime}$ and $\mathbf{x}_{2}^{\prime}$ in $E_{B}$, respectively, and their expressions are described as

$$
\begin{aligned}
& \mathbf{x}_{1}^{\prime}=\left\{\begin{array}{c}
r \cos \varphi_{1} \\
r \sin \varphi_{1} \\
l_{0}
\end{array}\right\}, \\
& \mathbf{x}_{2}^{\prime}=\left\{\begin{array}{c}
r \cos \varphi_{2} \\
r \sin \varphi_{2} \\
-l_{0}
\end{array}\right\} .
\end{aligned}
$$

By applying the method of the rotation matrix $\mathbf{R}_{1}^{\mathrm{T}}$, the coordinates of ER 1 and ER 2 are gained in $E_{I}$ (see (A.1)). Assuming that coordinates of the center of mass $G$ are 
$\mathbf{x}_{G}=\{x, y, 0\}^{\mathrm{T}}$ in $E_{I}$, the coordinates of ER 1 and ER 2 in $E_{I}$ can be expressed as follows:

$$
\begin{aligned}
& \mathbf{x}_{1}=\mathbf{x}_{G}+\mathbf{R}_{1}^{\mathrm{T}} \mathbf{x}_{1}^{\prime}, \\
& \mathbf{x}_{2}=\mathbf{x}_{G}+\mathbf{R}_{1}^{\mathrm{T}} \mathbf{x}_{2}^{\prime} .
\end{aligned}
$$

The kinetic energy $T$ of system is

$$
\begin{aligned}
T= & \frac{1}{2} m\left(\dot{x}^{2}+\dot{y}^{2}\right)+\frac{1}{2}\left(J_{1} \dot{\psi}^{2}+J_{2} \dot{\theta}^{2}\right)+\frac{1}{2} m_{1} \dot{\mathbf{x}}_{1}^{\mathrm{T}} \dot{\mathbf{x}}_{1} \\
& +\frac{1}{2} m_{2} \dot{\mathbf{x}}_{2}^{\mathrm{T}} \dot{\mathbf{x}}_{2}+\frac{1}{2} j_{01} \dot{\varphi}_{1}^{2}+\frac{1}{2} j_{02} \dot{\varphi}_{2}^{2},
\end{aligned}
$$

where $m$ is the mass of the rigid body; $m_{i}$ are the masses of ER $i$, respectively; $J_{i}$ are moments of inertia of the rigid body with rotating about $x$-axis and $y$-axis, respectively; $j_{0 i}$ are moment of inertia of two motors' rotors, respectively; $(\dot{\bullet})$ denotes $\mathrm{d} \cdot / \mathrm{d} t, i=1,2$.

In fact, the translational and rotational displacement of the rigid body are very small in the vibration condition. So the rotation matrix $\mathbf{R}_{1}^{\mathrm{T}}$ (see (A.2)) can be simplified into $\mathbf{R}_{2}^{\mathrm{T}}$, and the kinetic energy of the vibration system $T$ can also be simplified.

As illustrated in Figure 1(a), four springs have the same physical characteristics. If the vibration system does not run, the coordinates of the connection points between the springs and the rigid body overlap for $E_{I}$ and $E_{B}$ :

$$
\begin{aligned}
& \mathbf{x}_{k 10}=\mathbf{x}_{k 1}^{\prime \prime}=\left\{\begin{array}{c}
l_{x} \\
0 \\
l_{z}
\end{array}\right\}, \\
& \mathbf{x}_{k 20}=\mathbf{x}_{k 2}^{\prime \prime}=\left\{\begin{array}{c}
-l_{x} \\
0 \\
l_{z}
\end{array}\right\}, \\
& \mathbf{x}_{k 30}=\mathbf{x}_{k 3}^{\prime \prime}=\left\{\begin{array}{c}
l_{x} \\
0 \\
-l_{z}
\end{array}\right\}, \\
& \mathbf{x}_{k 40}=\mathbf{x}_{k 4}^{\prime \prime}=\left\{\begin{array}{c}
-l_{x} \\
0 \\
-l_{z}
\end{array}\right\} .
\end{aligned}
$$

So the coordinates of the connected points $(i=1,2,3,4)$ in $E_{I}$ can be expressed in the form

$$
\mathbf{x}_{k i}=\mathbf{x}_{G}+\mathbf{R}_{2}^{\mathrm{T}} \mathbf{x}_{k i}^{\prime \prime} .
$$

Then the potential energy $V$ of the vibration system is

$$
V=\frac{1}{2} \sum_{i=1}^{4}\left(\mathbf{x}_{k i}-\mathbf{x}_{k i 0}\right)^{\mathrm{T}} \mathbf{K}_{i}\left(\mathbf{x}_{k i}-\mathbf{x}_{k i 0}\right),
$$

where $\mathbf{K}_{i}=\operatorname{diag}\left(k_{x} / 4, k_{y} / 4,0\right)$ are stiffness matrix of spring $i$.
The viscous dissipation function $D$ of the vibration system is

$$
D=\frac{1}{2} \sum_{i=1}^{4} \dot{\mathbf{x}}_{k i}^{\mathrm{T}} \mathbf{F}_{i} \dot{\mathbf{x}}_{k i}
$$

where $\mathbf{F}_{i}=\operatorname{diag}\left(f_{x} / 4, f_{y} / 4,0\right)$ are damping matrix of spring $i$.

By using Lagrange equation, the motion equations are

$$
\frac{\mathrm{d}}{\mathrm{d} t} \frac{\partial(T-V)}{\partial \dot{q}_{i}}-\frac{\partial(T-V)}{\partial q_{i}}+\frac{\partial D}{\partial \dot{q}_{i}}=Q_{i}
$$

where $q_{i}$ are the generalized coordinates and $Q_{i}$ are the generalized forces of the vibration system.

The generalized coordinates are $\mathbf{q}=\left[x, y, \psi, \theta, \varphi_{1}, \varphi_{2}\right]^{\mathrm{T}}$, and the generalized forces are $Q_{x}=Q_{y}=Q_{\psi}=Q_{\theta}=0$, $Q_{\varphi 1}=T_{e 1}$, and $Q_{\varphi 2}=T_{e 2}$, in which $T_{e 1}$ and $T_{e 2}$ are the electromagnetic torques of motor 1 and motor 2, respectively.

Use (3), (6), and (7) and apply Lagrange equation (8) to obtain the equations of motion of the vibration system as follows [9-14]:

$$
\begin{aligned}
& M \ddot{x}+f_{x} \dot{x}+k_{x} x=\sum_{i=1}^{2} m_{i} r\left(\ddot{\varphi}_{i} \sin \varphi_{i}+\dot{\varphi}_{i}^{2} \cos \varphi_{i}\right), \\
& M \ddot{y}+f_{y} \dot{y}+k_{y} y=\sum_{i=1}^{2} m_{i} r\left(\dot{\varphi}_{i}^{2} \sin \varphi_{i}-\ddot{\varphi} \cos \varphi_{i}\right), \\
& J_{\psi} \ddot{\psi}+f_{\psi} \dot{\psi}+k_{\psi} \psi \\
& \quad=\sum_{i=1}^{2}(-1)^{i} m_{i} r l_{0}\left(\dot{\varphi}_{i}^{2} \sin \varphi_{i}-\ddot{\varphi}_{i} \cos \varphi_{i}\right), \\
& J_{\theta} \ddot{\theta}+f_{\theta} \dot{\theta}+k_{\theta} \theta \\
& \quad=\sum_{i=1}^{2}(-1)^{i+1} m_{i} r l_{0}\left(\dot{\varphi}_{i}^{2} \cos \varphi_{i}+\ddot{\varphi}_{i} \sin \varphi_{i}\right), \\
& J_{01} \ddot{\varphi}_{1}+f_{1} \dot{\varphi}_{1} \\
& \quad=T_{e 1}-m_{1} r\left[\cos \varphi_{1}\left(\ddot{y}-l_{0} \ddot{\psi}\right)-\sin \varphi_{1}\left(\ddot{x}+l_{0} \ddot{\theta}\right)\right], \\
& J_{02} \ddot{\varphi}_{2}+f_{2} \dot{\varphi}_{2} \\
& \quad=T_{e 2}-m_{2} r\left[\cos \varphi_{2}\left(\ddot{y}+l_{0} \ddot{\psi}\right)-\sin \varphi_{2}\left(\ddot{x}-l_{0} \ddot{\theta}\right)\right],
\end{aligned}
$$

where $J_{\psi}=J_{1}+\sum_{i=1}^{2} m_{i} l_{0}^{2}$ and $J_{\theta}=J_{2}+\sum_{i=1}^{2} m_{i} l_{0}^{2}, k_{\psi}=k_{y} l_{z}^{2}$ and $k_{\theta}=k_{x} l_{z}^{2}, f_{\psi}=f_{y} l_{z}^{2}$ and $f_{\theta}=f_{x} l_{z}^{2}, M=m+m_{1}+m_{2}$, and $J_{0 i}=j_{0 i}+m_{i} r^{2}$ are moment of inertia of ERs, $f_{1}$ and $f_{2}$ are damping coefficient of the axis of motor, and $(\bullet)$ denotes $\mathrm{d}^{2}(\bullet) / \mathrm{d} t^{2}$. 


\section{Synchronization of Two ERs}

Assuming that the average phase of two ERs is $\varphi$, the phase difference between two ERs is $2 \alpha$ :

$$
\begin{aligned}
& \varphi_{1}=\varphi+\alpha, \\
& \varphi_{2}=\varphi-\alpha .
\end{aligned}
$$

Since the response of the vibration system is periodic, the changes of angular velocities $\dot{\varphi}$ of two motors are periodic as well. Assuming the lowest common multiple of period between two ERs is $T_{0}$, the average value of $\dot{\varphi}$ over $T_{0}$ must be constant; that is,

$$
\omega_{0}=\frac{1}{T_{0}} \int_{0}^{T_{0}} \omega(t) \mathrm{d} t=\text { constant }
$$

We assume that the synchronization velocity between two motors is $\omega_{m 0}$, and the instantaneous change coefficients of $\dot{\varphi}$ and $\alpha$ are $\varepsilon_{1}$ and $\varepsilon_{2}$, respectively; then we obtain

$$
\begin{aligned}
& \dot{\varphi}_{1}=\left(1+\varepsilon_{1}+\varepsilon_{2}\right) \omega_{m 0}, \\
& \dot{\varphi}_{2}=\left(1+\varepsilon_{1}-\varepsilon_{2}\right) \omega_{m 0} .
\end{aligned}
$$

Substituting (10) into the first four formulas of (9), we obtain the responses of the steady state of the vibration system as follows [9-11]:

$$
\begin{aligned}
& x=-\frac{r r_{m}}{\mu_{x}}\left[\cos \left(\varphi+\alpha+\gamma_{x}\right)+\eta \cos \left(\varphi-\alpha+\gamma_{x}\right)\right], \\
& y=-\frac{r r_{m}}{\mu_{y}}\left[\sin \left(\varphi+\alpha+\gamma_{y}\right)+\eta \sin \left(\varphi-\alpha+\gamma_{y}\right)\right], \\
& \psi \\
& =-\frac{r r_{m} r_{\psi}}{\mu_{\psi} l_{\psi}}\left[-\sin \left(\varphi+\alpha+\gamma_{\psi}\right)+\eta \sin \left(\varphi-\alpha+\gamma_{\psi}\right)\right], \\
& \theta=-\frac{r r_{m} r_{\theta}}{\mu_{\theta} l_{\theta}}\left[\cos \left(\varphi+\alpha+\gamma_{\theta}\right)+\eta \cos \left(\varphi-\alpha+\gamma_{\theta}\right)\right],
\end{aligned}
$$

where

$$
\begin{aligned}
& \omega_{x}=\sqrt{\frac{k_{x}}{M}}, \\
& \omega_{y}=\sqrt{\frac{k_{y}}{M}}, \\
& \omega_{\psi}=\sqrt{\frac{k_{\psi}}{J_{\psi}}} \\
& \omega_{\theta}=\sqrt{\frac{k_{\theta}}{J_{\theta}}} \\
& \xi_{y}=\frac{f_{y}}{2 \sqrt{M k_{y}}}, \\
& \xi_{x}=\frac{f_{x}}{2 \sqrt{M k_{x}}},
\end{aligned}
$$

$$
\begin{aligned}
\xi_{\psi} & =\frac{f_{\psi}}{2 \sqrt{J_{\psi} k_{\psi}}}, \\
\xi_{\theta} & =\frac{f_{\theta}}{2 \sqrt{J_{\theta} k_{\theta}}}, \\
r_{m} & =\frac{m_{0}}{M}, \\
m_{1} & =m_{0}, \\
m_{2} & =\eta m_{0}, \\
l_{\psi} & =\sqrt{\frac{J_{\psi}}{M}}, \\
l_{\theta} & =\sqrt{\frac{J_{\theta}}{M}}, \\
\gamma_{i} & =\arctan \frac{2 \xi_{i}}{1-\left(\omega_{i} / \omega_{m 0}\right)}, \\
r_{\psi} & =\frac{l_{0}}{l_{\psi}}, \\
r_{\theta} & =\frac{l_{0}}{l_{\theta}}, \\
\mu_{i} & =1-\left(\frac{\omega_{i}}{\omega_{m 0}}\right)^{2}, y, \psi, \theta .
\end{aligned}
$$

$\omega_{x}, \omega_{y}, \omega_{\psi}$, and $\omega_{\theta}$ are the natural frequency of the vibration system in $x-, y-, \psi_{-}$, and $\theta$-directions; $f_{x}, f_{y}, f_{\psi}$, and $f_{\theta}$ are the corresponding damping ratios of the springs in $x_{-}^{-}, y_{-}, \psi_{-}$, and $\theta$-directions; $\pi-\gamma_{x}, \pi-\gamma_{y}, \pi-\gamma_{\psi}$, and $\pi-\gamma_{\theta}$ are phase angle in $x-, y-, \psi^{-}$, and $\theta$-directions, respectively; $r_{m}$ is mass ratio between the mass of ER 1 and the mass of the vibration system; $\eta$ is mass ratio between the mass of ER 1 and that of ER $2 ; r_{\psi}\left(r_{\theta}\right)$ is defined as the ratio between $l_{0}$ and $l_{\psi}\left(l_{\theta}\right) ; l_{\psi}$ and $l_{\theta}$ are the equivalent rotating radius of the vibration system about the $x$-axis and $y$-axis of the rigid body.

3.1. The Dimensionless Coupling Equation. Inserting $\ddot{x}, \ddot{y}, \ddot{\psi}$, and $\ddot{\theta}$ (with respect to time $t$ ) into the last two formulas of (9) and integrating them over $\varphi=0 \sim 2 \pi$, respectively, we have

$$
\begin{aligned}
& J_{01} \omega_{m 0}\left(\dot{\bar{\varepsilon}}_{1}+\dot{\bar{\varepsilon}}_{2}\right)+f_{1} \omega_{m 0}\left(1+\bar{\varepsilon}_{1}+\bar{\varepsilon}_{2}\right)=T_{e 1}-T_{L 1}, \\
& J_{02} \omega_{m 0}\left(\dot{\bar{\varepsilon}}_{1}-\dot{\bar{\varepsilon}}_{2}\right)+f_{2} \omega_{m 0}\left(1+\bar{\varepsilon}_{1}-\bar{\varepsilon}_{2}\right)=T_{e 2}-T_{L 2}
\end{aligned}
$$

with

$$
\begin{aligned}
& \bar{T}_{L 1}=\chi_{11}^{\prime} \dot{\bar{\varepsilon}}_{1}+\chi_{12}^{\prime} \dot{\bar{\varepsilon}}_{2}+\chi_{11} \bar{\varepsilon}_{1}+\chi_{12} \bar{\varepsilon}_{2}+\chi_{a}+\chi_{f 1}, \\
& \bar{T}_{L 2}=\chi_{21}^{\prime} \dot{\bar{\varepsilon}}_{1}+\chi_{21}^{\prime} \dot{\bar{\varepsilon}}_{2}+\chi_{21} \bar{\varepsilon}_{1}+\chi_{22} \bar{\varepsilon}_{2}-\chi_{a}+\chi_{f 2} .
\end{aligned}
$$


The coefficients of $\bar{\varepsilon}_{1}, \bar{\varepsilon}_{2}, \dot{\bar{\varepsilon}}_{1}$, and $\dot{\bar{\varepsilon}}_{2}$ in (16) are listed in Appendix B. Based on the direct motions separation [911], $\varepsilon_{1}, \varepsilon_{2}, \dot{\varepsilon}_{1}, \dot{\varepsilon}_{2}$, and $\alpha$ are considered to be their integral midvalves $\bar{\varepsilon}_{1}, \bar{\varepsilon}_{2}, \dot{\bar{\varepsilon}}_{1}, \dot{\bar{\varepsilon}}_{2}$, and $\bar{\alpha}$, respectively.

According to the electromagnetic torques formula of induction motor, we have [23]

$$
\begin{aligned}
& T_{e 1}=T_{e 01}-k_{e 01}\left(\bar{\varepsilon}_{1}+\bar{\varepsilon}_{2}\right), \\
& T_{e 2}=T_{e 02}-k_{e 02}\left(\bar{\varepsilon}_{1}-\bar{\varepsilon}_{2}\right),
\end{aligned}
$$

where

$$
\begin{aligned}
T_{e 0 i} & =\frac{k\left(\omega_{s i}-\omega_{0}\right)}{a_{2} \omega_{0}^{2}+a_{1} \omega_{0}+a_{0}}, \\
k_{e 0 i} & =\frac{k\left[-a_{2} \omega_{0}^{3}+2 a_{2} \omega_{s i} \omega_{0}^{2}+\left(a_{1} \omega_{s i}+a_{0}\right) \omega_{0}\right]}{\left(a_{2} \omega_{0}^{2}+a_{1} \omega_{0}+a_{0}\right)^{2}}, \\
k & =\frac{3 n_{p} L_{m}^{2} U^{2}}{R_{s}^{2} R_{r}}, \\
T_{s} & =\frac{L_{s}}{R_{s}}, \\
T_{r} & =\frac{L_{r}}{R_{r}}, \\
\sigma & =1-\frac{L_{m}^{2}}{L_{s} L_{r}}, \\
\omega_{0} & =n_{p} \omega_{m 0}, \\
a_{0} & =\left(1-\omega_{s i}^{2} T_{s} T_{r} \sigma\right)^{2}+\omega_{s i}^{2}\left(T_{r}+T_{s}\right)^{2}, \\
a_{1} & =2\left(1-\omega_{s i}^{2} T_{s i} T_{r} \sigma\right) \omega_{s i} T_{s} T_{r} \sigma-2 \omega_{s i}\left(T_{r}+T_{s i}\right) T_{r} \\
a_{2} & =T_{r}^{2}+\omega_{s i}^{2} T_{s i}^{2} T_{r}^{2} \sigma^{2}
\end{aligned}
$$

$$
i=1,2 \text {. }
$$

$n_{p}$ is the number of pole pairs; $L_{s}$ is the stator inductance, $L_{r}$ is the rotor inductance, and $L_{m}$ is the mutual inductance; $R_{s}$ is the stator resistance and $R_{r}$ is the rotor resistance; $\omega_{s i}$ is the synchronization electric angular velocity and $\omega$ is the rotor electric angular velocity; $U$ is the phase voltage.

Substituting (16) and (17) into (15), we obtain the dimensionless coupling equations of two ERs as follows:

$$
\mathrm{A} \dot{\bar{\varepsilon}}=\mathbf{B} \bar{\varepsilon}+\mathbf{u}
$$

where $\overline{\boldsymbol{\varepsilon}}=\left\{\begin{array}{ll}\bar{\varepsilon}_{1} & \bar{\varepsilon}_{2}\end{array}\right\}^{\mathrm{T}}, \mathbf{A}$ and $\mathbf{B}$ are $2 \times 2$ matrices, $\mathbf{A}=\left[\begin{array}{ll}a_{11} & a_{12} \\ a_{21} & a_{22}\end{array}\right]$, $\mathbf{B}=-\omega_{m 0}\left[\begin{array}{ll}b_{11} & b_{12} \\ b_{21} & b_{22}\end{array}\right], \mathbf{u}=\left[\begin{array}{l}u_{1} \\ u_{2}\end{array}\right]$,

$$
\begin{aligned}
u_{1}= & \frac{T_{e 01}}{m_{0} r^{2} \omega_{m 0}}+\frac{T_{e 02}}{m_{0} r^{2} \omega_{m 0}}-\frac{f_{1}+f_{2}}{m_{0} r^{2}} \\
& -\frac{\omega_{m 0}}{2} W_{s 0}\left(1+\eta^{2}\right)-\omega_{m 0} W_{s} \cos 2 \bar{\alpha}
\end{aligned}
$$

$$
\begin{aligned}
u_{2}= & \frac{T_{e 01}}{m_{0} r^{2} \omega_{m 0}}-\frac{T_{e 02}}{m_{0} r^{2} \omega_{m 0}}-\frac{f_{1}-f_{2}}{m_{0} r^{2}} \\
& -\frac{\omega_{m 0}}{2} W_{s 0}\left(1-\eta^{2}\right)-\omega_{m 0} W_{c} \sin 2 \bar{\alpha}, \\
\rho_{1}= & 1-\frac{W_{c 0}}{2}, \\
\rho_{2}= & \eta-\eta^{2} \frac{W_{c 0}}{2}, \\
\kappa_{1}= & \frac{k_{e 01}}{m_{0} r^{2} \omega_{m 0}^{2}}+\frac{f_{1}}{m_{0} r^{2} \omega_{m 0}}+W_{s 0}, \\
\kappa_{2}= & \frac{k_{e 02}}{m_{0} r^{2} \omega_{m 0}^{2}}+\frac{f_{2}}{m_{0} r^{2} \omega_{m 0}}+\eta W_{s 0}, \\
a_{11}= & \rho_{1}+\rho_{2}+W_{c} \cos 2 \bar{\alpha}, \\
a_{22}= & \rho_{1}+\rho_{2}-W_{c} \cos 2 \bar{\alpha}, \\
a_{12}= & \rho_{1}-\rho_{2}+W_{s} \sin 2 \bar{\alpha}, \\
a_{21}= & \rho_{1}-\rho_{2}-W_{s} \sin 2 \bar{\alpha}, \\
b_{11}= & \kappa_{1}+\kappa_{2}-2 W_{s} \cos 2 \bar{\alpha}, \\
b_{22}= & \kappa_{1}+\kappa_{2}+2 W_{s} \cos 2 \bar{\alpha}, \\
b_{12}= & \kappa_{1}-\kappa_{2}-2 W_{c} \sin 2 \bar{\alpha}, \\
b_{21}= & \kappa_{1}-\kappa_{2}+2 W_{c} \sin 2 \bar{\alpha} . \\
&
\end{aligned}
$$

3.2. The Synchronization Condition. Substituting $\bar{\varepsilon}_{1}=0$ and $\bar{\varepsilon}_{2}=0$ into (19), we obtain $u_{1}=0$ and $u_{2}=0$. According to $u_{2}=0$, we have

$$
2 \bar{\alpha}=\arcsin \frac{T_{D}}{T_{C}},
$$

where $T_{C}$ is called the synchronization torque, $T_{C}=$ $m_{0} r^{2} \omega_{m 0}^{2} W_{c} ; T_{D}$ is called the difference of the residual electromagnetic torques between the two motors, $T_{D}=T_{R 1}$ $T_{R 2} ; T_{R 1}=T_{e 01}-f_{1} \omega_{m 0}-m_{0} r^{2} \omega_{m 0}^{2} W_{s 0} / 2$ and $T_{R 2}=T_{e 02}-$ $f_{2} \omega_{m 0}-m_{0} r^{2} \omega_{m 0}^{2} \eta^{2} W_{s 0} / 2$.

Since $|\sin 2 \bar{\alpha}| \leq 1$, the synchronization condition that the vibration system carries out the synchronized motion of two ERs is

$$
T_{C} \geq\left|T_{D}\right| \text {. }
$$

Hence, the synchronization condition of two ERs achieving the synchronized motion is that the synchronization torque $T_{C}$ is greater than or equal to the absolute value of the difference between the residual electromagnetic torques of two motors $T_{D}$.

We define ratio value between the synchronization torque $T_{C}$ and the load torque $T_{L}$ as the coefficient of synchronization ability, $\zeta$, as follows:

$$
\zeta=\left|\frac{T_{C}}{T_{L}}\right|=\frac{2\left|W_{c}\right|}{\left|\left(1+\eta^{2}\right) W_{s 0}+2 W_{s} \cos 2 \bar{\alpha}\right|},
$$


where $T_{L}$ is the load torque that the vibration system acts on two motors, $T_{L}=m_{0} r^{2} \omega_{m 0}^{2} W_{s 0}\left[\left(1+\eta^{2}\right) W_{s 0}+2 W_{s} \cos 2 \bar{\alpha}\right] / 2$. The greater the coefficient of synchronization ability, the stronger the synchronization ability of the vibration system and the easier to carry out synchronized motion of two motors.

3.3. Stability of the Synchronized Motion. If the vibration system satisfies the synchronization condition, we solve $u_{1}=$ 0 and $u_{2}=0$ for $\omega_{m 0}$ and $\bar{\alpha}$, which are denoted by $\omega_{m 0}^{*}$ and $\bar{\alpha}_{0}$, respectively. Linearize (19) around $\bar{\alpha}=\bar{\alpha}_{0}$, consider $\Delta \dot{\bar{\alpha}}=\bar{\alpha}-\bar{\alpha}_{0}=\omega_{m 0}^{*} \bar{\varepsilon}_{2}$, use the notation $\mathbf{z}=\left\{\begin{array}{lll}\bar{\varepsilon}_{1} & \bar{\varepsilon}_{2} & \Delta \bar{\alpha}\end{array}\right\}^{\mathrm{T}}$, and write the dimensionless coupling equation as follows:

$$
\dot{\mathbf{z}}=\mathbf{C} \mathbf{z},
$$

where

$$
\begin{aligned}
\mathbf{C} & =\mathbf{A}^{\prime-1} \mathbf{B}^{\prime}, \\
\mathbf{A}^{\prime} & =\left[\begin{array}{ccc}
a_{11} & a_{12} & 0 \\
a_{21} & a_{22} & 0 \\
0 & 0 & 1
\end{array}\right], \\
\mathbf{B}^{\prime} & =-\omega_{m 0}\left[\begin{array}{ccc}
b_{11} & b_{12} & -2 W_{s} \sin 2 \bar{\alpha}_{0} \\
b_{21} & b_{22} & 2 W_{c} \cos 2 \bar{\alpha}_{0} \\
0 & -1 & 0
\end{array}\right] .
\end{aligned}
$$

Assuming $\mathbf{z}=\mathbf{v} \exp (\lambda t)$ in the form of exponential timedependent, we deduce the characteristic equation for the eigenvalue $\lambda$ by solving the determinant equation $\operatorname{det}(\mathbf{C}-$ $\lambda \mathbf{I})=0$, as follows:

$$
\lambda^{3}+c_{1} \lambda^{2}+c_{2} \lambda+c_{3}=0
$$

where $c_{1}=4 \omega_{m 0} H_{1} / H_{0}, c_{2}=2 \omega_{m 0}^{2} H_{2} / H_{0}, c_{3}=2 \omega_{m 0}^{3} H_{3} / H_{0}$, and

$$
\begin{aligned}
H_{0}= & 4 \rho_{1} \rho_{2}-W_{c}^{2} \cos ^{2} 2 \alpha_{0}+W_{s}^{2} \sin ^{2} 2 \alpha_{0}, \\
H_{1}= & \rho_{1} \kappa_{2}+\rho_{2} \kappa_{1}-W_{s} W_{c}, \\
H_{2}= & 2 \kappa_{1} \kappa_{2}+\left(\rho_{1}+\rho_{2}\right) W_{c} \cos 2 \alpha_{0} \\
& +\left(\rho_{1}-\rho_{2}\right) W_{s} \sin 2 \alpha_{0}+W_{c}^{2}\left(1+\sin ^{2} 2 \alpha_{0}\right) \\
& -W_{s}^{2}\left(1+\cos ^{2} 2 \alpha_{0}\right), \\
H_{3}= & \left(\kappa_{1}+\kappa_{2}\right) W_{c} \cos 2 \alpha_{0}+\left(\kappa_{1}-\kappa_{2}\right) W_{s} \sin 2 \alpha_{0} \\
& +2 W_{s} W_{c} .
\end{aligned}
$$

In engineering, because the maximum of damping constant of the vibration systems is very small [9-11], $W_{s}$ can be neglected with comparing $W_{c}$ in $c_{1}, c_{2}$, and $c_{3}$. Then, we simplify $H_{i}(i=0,1,2,3)$ as follows:

$$
\begin{aligned}
& H_{0}^{\prime}=4 \rho_{1} \rho_{2}-W_{c}^{2} \cos ^{2} 2 \alpha_{0}, \\
& H_{1}^{\prime}=\rho_{1} \kappa_{2}+\rho_{2} \kappa_{1},
\end{aligned}
$$

$$
\begin{aligned}
H_{2}^{\prime}= & 2 \kappa_{1} \kappa_{2}+\left(\rho_{1}+\rho_{2}\right) W_{c} \cos 2 \alpha_{0} \\
& +W_{c}^{2}\left(1+\sin ^{2} 2 \alpha_{0}\right), \\
H_{3}^{\prime}= & \left(\kappa_{1}+\kappa_{2}\right) W_{c} \cos 2 \alpha_{0} .
\end{aligned}
$$

Applying Routh-Hurwitz criterion [9-11], we deduce the stability condition of the synchronized motion of two ERs when the trivial solution $z_{i}=0$ is stable, as follows:

$$
\begin{aligned}
c_{1} & >0, \\
c_{3} & >0, \\
c_{1} c_{2} & >c_{3} .
\end{aligned}
$$

Based on the sign of $H_{0}^{\prime}$, we rewrite inequality (29) as inequalities (30) and (31) as follows:

$$
\begin{aligned}
H_{0}^{\prime} & >0, \\
H_{1}^{\prime} & >0, \\
H_{3}^{\prime} & >0, \\
4 H_{1}^{\prime} H_{2}^{\prime}-H_{0}^{\prime} H_{3}^{\prime} & >0, \\
H_{0}^{\prime} & <0, \\
H_{1}^{\prime} & <0, \\
H_{3}^{\prime} & <0, \\
4 H_{1}^{\prime} H_{2}^{\prime}-H_{0}^{\prime} H_{3}^{\prime} & >0 .
\end{aligned}
$$

Since $\kappa_{1}>0$ and $\kappa_{2}>0$, we can obtain from $H_{0}^{\prime}>0$ and $H_{1}^{\prime}>0$,

$$
\begin{aligned}
\rho_{1} & >0, \\
\rho_{2} & >0, \\
4 \rho_{1} \rho_{2}-W_{c}^{2} \cos ^{2} 2 \alpha_{0} & >0 .
\end{aligned}
$$

According to $H_{3}^{\prime}>0$, we obtain

$$
W_{c} \cos 2 \alpha_{0}>0 \text {. }
$$

Substituting the expressions of $H_{i}^{\prime}(i=0,1,2,3)$ into $4 H_{1}^{\prime} H_{2}^{\prime}-$ $H_{0}^{\prime} H_{3}^{\prime}>0$,

$$
\begin{aligned}
& 4 W_{c}(\left.\kappa_{2} \rho_{1}^{2}+\kappa_{1} \rho_{2}^{2}\right) \cos 2 \alpha_{0}+\left(\kappa_{1}+\kappa_{2}\right) W_{c}^{3} \cos ^{3} 2 \alpha_{0} \\
&+4\left(\kappa_{2} \rho_{1}+\kappa_{1} \rho_{2}\right)\left(W_{c}^{2}+2 \kappa_{1} \kappa_{2}+W_{c}^{2} \sin ^{2} 2 \alpha_{0}\right)
\end{aligned}
$$

$>0$.

When $\rho_{1} \kappa_{2}+\rho_{2} \kappa_{1}>0$, inequality (34) is true. Hence, inequalities (32) and (33) satisfy inequality (34).

If $H_{0}^{\prime}<0, H_{1}^{\prime}<0$ needs $\rho_{1} \kappa_{2}+\rho_{2} \kappa_{1}<0$ and $H_{3}^{\prime}<0$ needs $W_{c} \cos 2 \alpha_{0}<0$. However, the left side of inequality (34) is false. Hence, $H_{0}^{\prime}<0, H_{1}^{\prime}<0$, and $H_{3}^{\prime}<0$ cannot satisfy the condition of $4 H_{1}^{\prime} H_{2}^{\prime}-H_{0}^{\prime} H_{3}^{\prime}>0$. 
In a word, inequality (30) satisfies Routh-Hurwitz criterion. So we obtain the stability condition of two ERs achieving the synchronized motion, which is $W_{c} \cos 2 \alpha_{0}>0$. If $W_{c}>0$, $2 \alpha_{0} \in\left(-90^{\circ}, 90^{\circ}\right)$ satisfies the stability condition, and if $W_{c}<$ $0,2 \alpha_{0} \in\left(90^{\circ}, 270^{\circ}\right)$ satisfies the stability condition. So the vibration system has two kinds of stable synchronized motion state when the synchronization condition is satisfied.

\section{Numerical Results and Discussions}

Some simplified forms of theoretical analysis for the synchronization problem have been given in previous section. This section will present numerical results to compare with theoretical analysis for the synchronization condition and the stability condition.

The dimensionless parameters of the vibration system, $W_{s}, W_{s 0}, W_{c}$, and $W_{c 0}$, have an effect on the synchronization condition and the stability condition and are also functions of $r_{m}, \eta, r_{\psi}, r_{\theta}, \mu_{x}, \mu_{y}, \mu_{\psi}$, and $\mu_{\theta}$. Since the motion trajectory of this type of vibration machine is assumed as circle in $x y$ plane, such as the vibration mill that achieves the spatial circular motion, we assume $k_{x}=k_{y}, f_{x}=f_{y}$, and $J_{\psi}=J_{\theta}$. However, $\mu_{x}, \mu_{y}, \mu_{\psi}$, and $\mu_{\theta}$ change little (24/25 99/100) that can be treated as constant [12]. Herein, we will study the function of dimensionless parameters $r_{m}, \eta$, and $r_{\psi}=r_{\theta}$ on the synchronization ability and the stability of synchronization.

The actual parameter values of the vibration system are $M=230 \mathrm{~kg}, m_{0}=7.6 \mathrm{~kg}, J_{\psi}=J_{\theta}=67 \mathrm{~kg} \cdot \mathrm{m}^{2}, k_{x}=k_{y}=$ $160 \mathrm{kN} / \mathrm{m}, k_{\psi}=k_{\theta}=47 \mathrm{kN} / \mathrm{rad}, f_{x}=f_{y}=270 \mathrm{kNs} / \mathrm{m}$, and $f_{\psi}=f_{\theta}=79 \mathrm{kNms} / \mathrm{rad}$. The parameters of the two motors are the same: $R_{s}=22.89 \Omega, R_{r}=13.34 \Omega, L_{s}=873 \mathrm{mH}$, $L_{r}=875 \mathrm{mH}$, and $L_{m}=830 \mathrm{mH}$.

We give the expression of $r_{\psi}$ versus $r_{m}$ and $\eta$ to reflect $r_{\psi}$ effects on the synchronization ability. Based on the expression of $r_{\psi}$, its maximum can be simplified in the form

$$
r_{\psi \max }^{2}=\lim _{l_{0} \rightarrow \infty} r_{\psi}^{2}=\frac{1+r_{m}(1+\eta)}{r_{m}(1+\eta)} .
$$

The synchronized motion of the vibration system is stable when it satisfies (33) and (34). Figure 2 shows that $r_{\psi \max } \approx 7$ for $\eta=1$; we will arrange the value of $r_{\psi}$ from 0 to 7 in next discussions.

According to the theoretical analysis in previous section, $T_{C} \geq\left|T_{D}\right|$, to satisfy the synchronization condition. When two identical motors drive the two different masses of ERs, we can obtain the simplified form of (22):

$$
W_{c} \geq\left|\frac{W_{s 0}\left(1-\eta^{2}\right)}{2}\right| \text {. }
$$

Figure 3 shows the regions of implementing the synchronized motion in $\eta r_{\psi}$-plane. We divide the $\eta r_{\psi}$-plane into three regions. The phase difference that the vibration system carries out during synchronized motion is $2 \alpha \in\left(-90^{\circ}, 90^{\circ}\right)$ in Region I, and it is $2 \alpha \in\left(90^{\circ}, 270^{\circ}\right)$ in Region II. The vibration system cannot carry out the synchronized motion in Regions I and II. Regions I, II, and III converge into a point $(\eta=1$ and $r_{\psi} \approx 1$ ), and $T_{D}=0$ at this point. We know that this

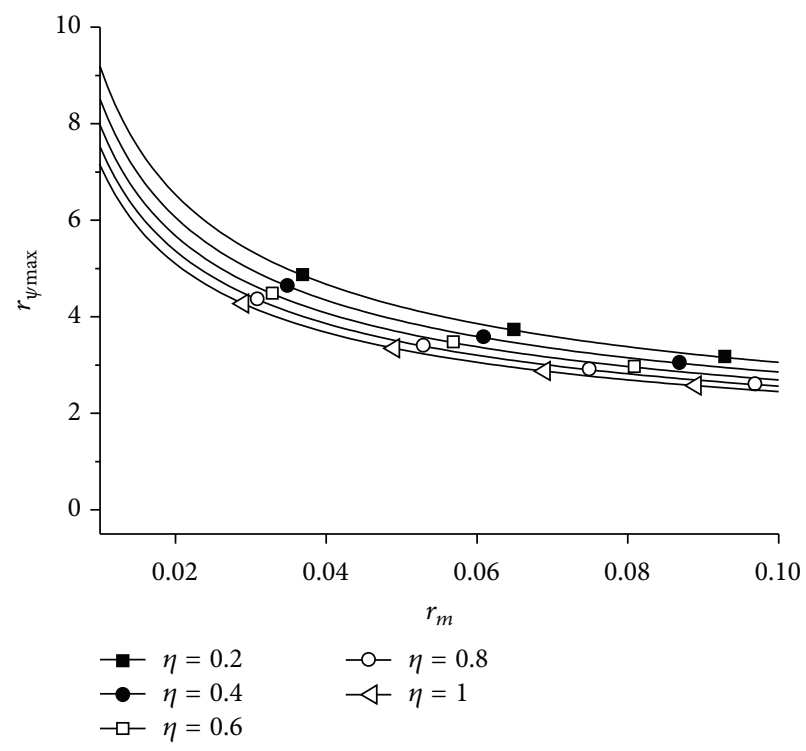

FIgURE 2: Maximum of $r_{\psi}$.

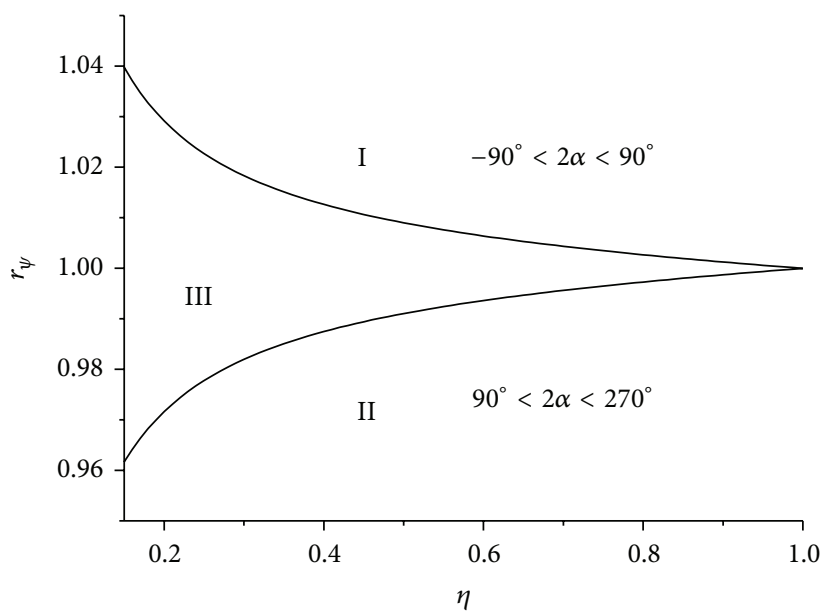

FIGURE 3: Regions of implementing synchronization.

point is the optimal parameters of two ERs to enhance the synchronization ability.

4.1. Implementing the Synchronized Motion. Since the dimensionless parameter $W_{c}$ is the function of $r_{\psi}$, its value changes with changing $r_{\psi}$ and has zero point. The synchronization torque $T_{C}$ is the function of $W_{c}$, so it has similar changes with $W_{c}$. As mentioned in theoretical analysis, the greater the values of the synchronization torque, the easier the two ERs to implement synchronization. Figure 4 shows the changes of $T_{C}$ with different mass ratio $\eta$ between ERs. $T_{C}$ has zero point in every case, and the point at about $r_{\psi}=1$ depends on structure parameters of the vibration system. According to the regions divided in Figure 3, the vibration system cannot implement the synchronized motion when $r_{\psi}=1$. $T_{C}$ obtains the maximal values at $\eta=1$, and the vibration system operates at its best synchronization state. In addition, the greater $r_{m}$, the greater $T_{C}$. Comparing with $r_{\psi}<1$, 


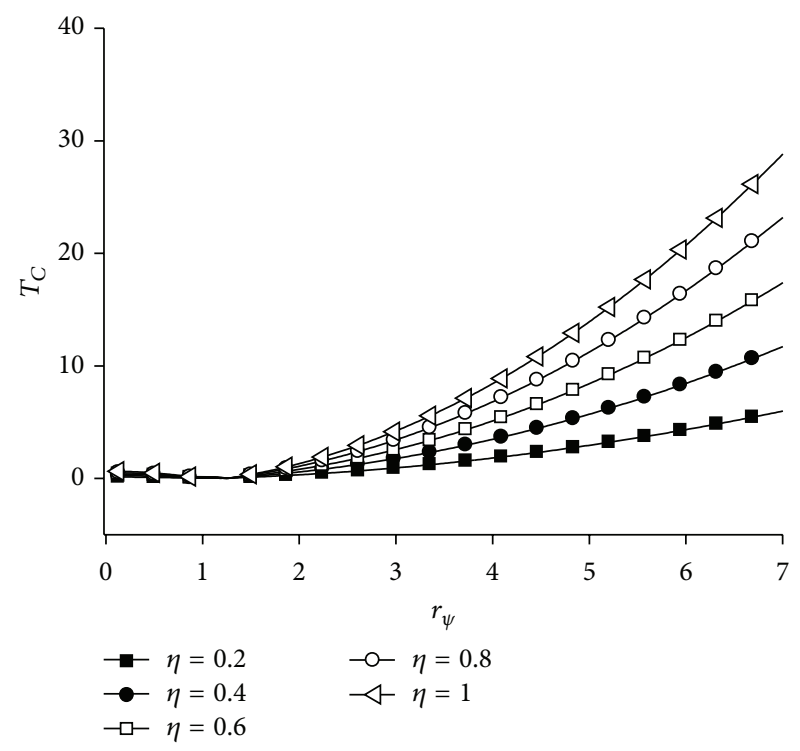

(a)

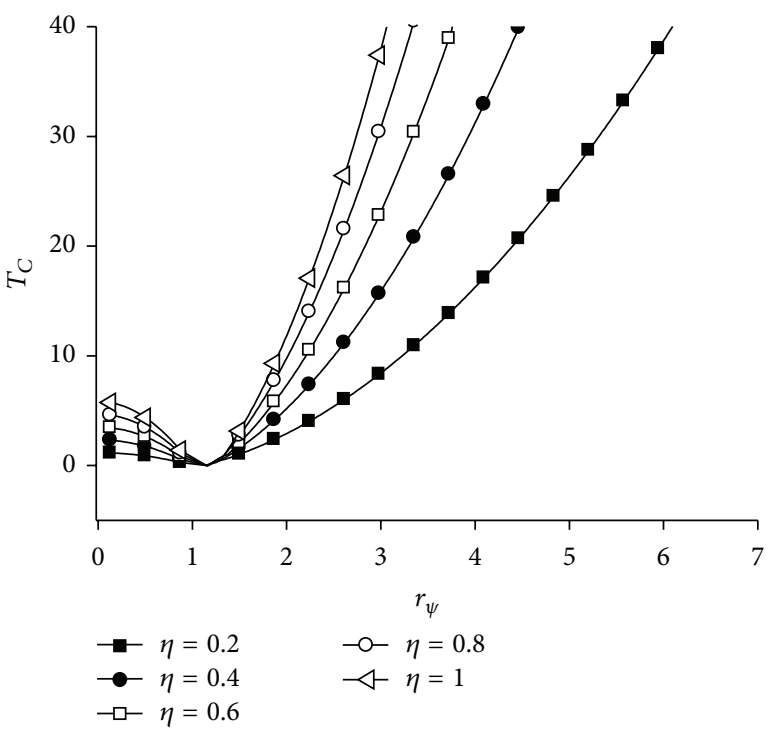

(b)

FIgURE 4: Synchronization torque: (a) $r_{m}=0.03$; (b) $r_{m}=0.09$.

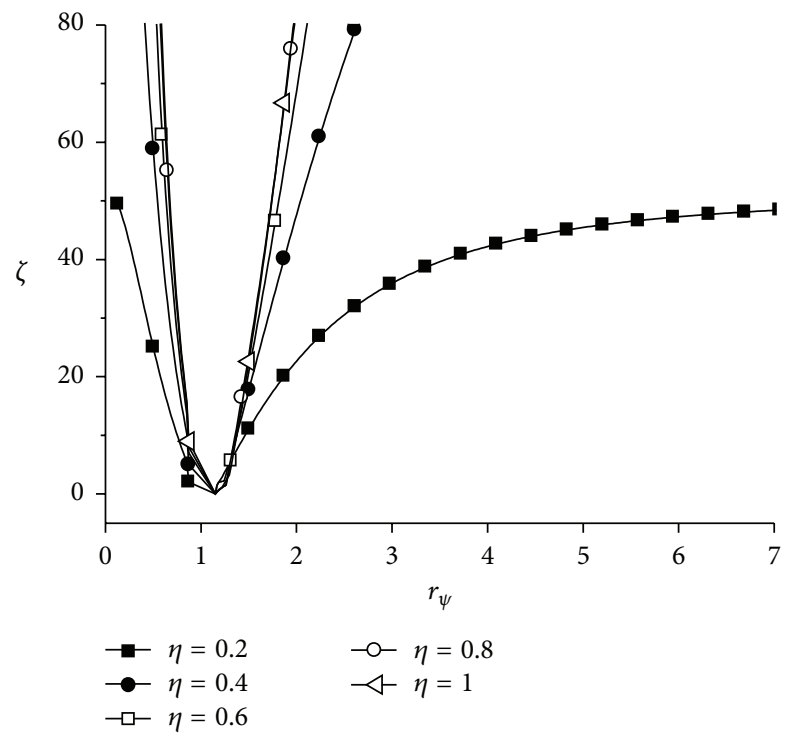

FIGURE 5: Coefficients of synchronization ability.

the vibration system can easier carry out the synchronized motion for $r_{\psi}>1$. When $r_{\psi}>1, T_{C}$ increases with increasing $r_{\psi}$. By comparing the synchronization torque with the different mass ratio, the better the symmetry of the structural parameter, the greater the synchronization torque.

Figure 5 shows the changes of $\zeta$ with different mass ratio $\eta$ between ERs. Since $\zeta$ is the function of $W_{c}, W_{s 0}$, and $W_{s}$ and they are the function of $r_{m}, r_{m}$ has no effect on $\zeta$. In addition, $\zeta$ has zero point for every $\eta$. From Figure 5, it can be seen that all $\zeta$ first decrease with increasing $r_{\psi}$ and then decrease. To sum up, $r_{\psi}$ plays a decisive role in the adjustment of the synchronization ability.

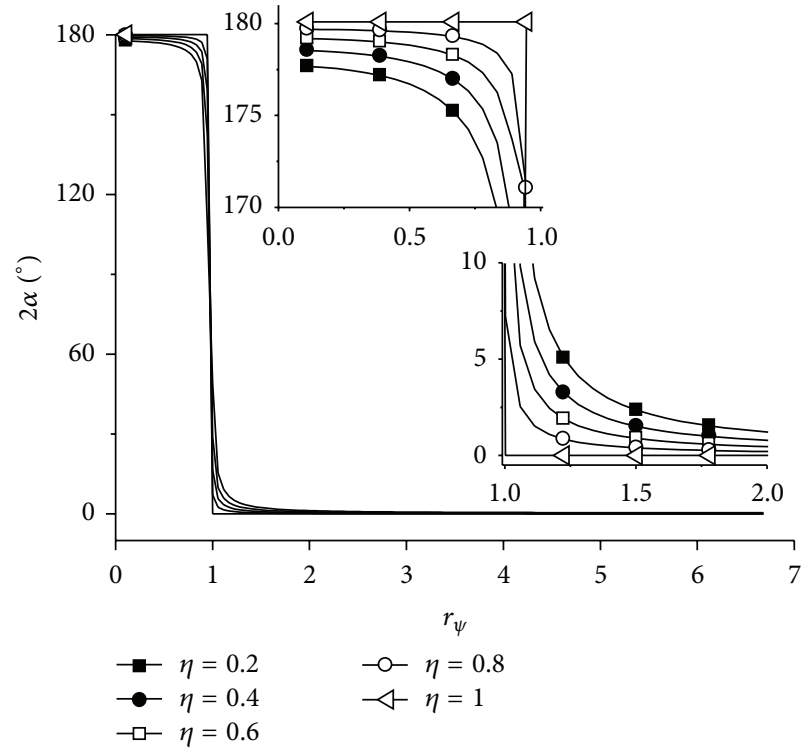

FIGURE 6: Approximate values of $2 \alpha$ versus $r_{\psi}$.

4.2. Stability of the Synchronized Motion. The stability condition of two ERs achieving the synchronized motion is $W_{c} \cos 2 \alpha_{0}>0$. When the stability condition is satisfied, $W_{c}$ determines the change of the phase difference $2 \alpha$. In addition, the phase difference determines the motion type of the vibration system. In other words, if the structural parameters of the vibration system satisfy the synchronization condition, the motion type is determined by $r_{\psi}$. This is the choosing motion characteristic that the motion type of the vibration system depends on its structural parameters.

Figure 6 shows the regions where the phase difference $2 \alpha$ changes with the different mass ratio $\eta$ between ERs. 


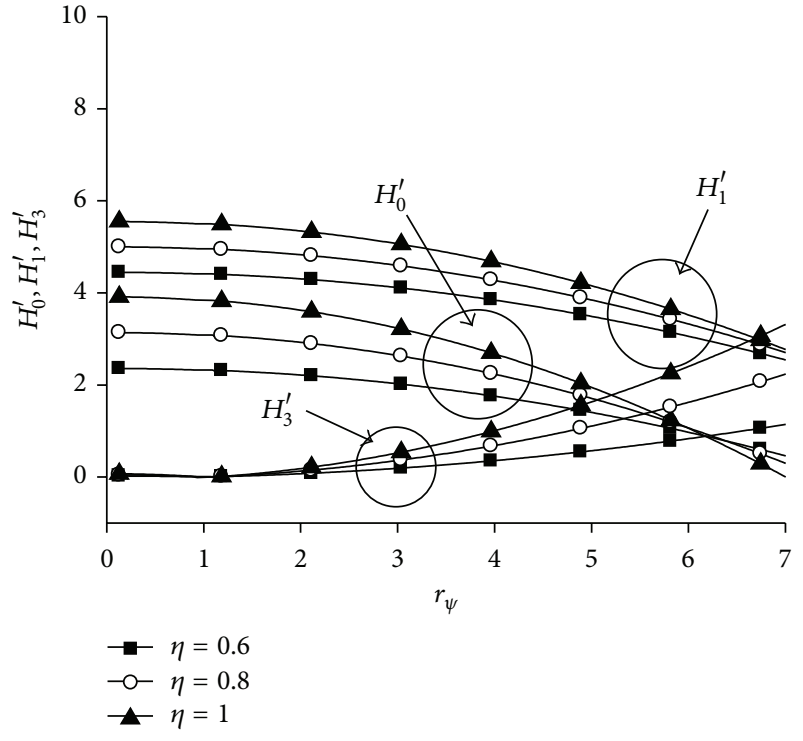

(a)

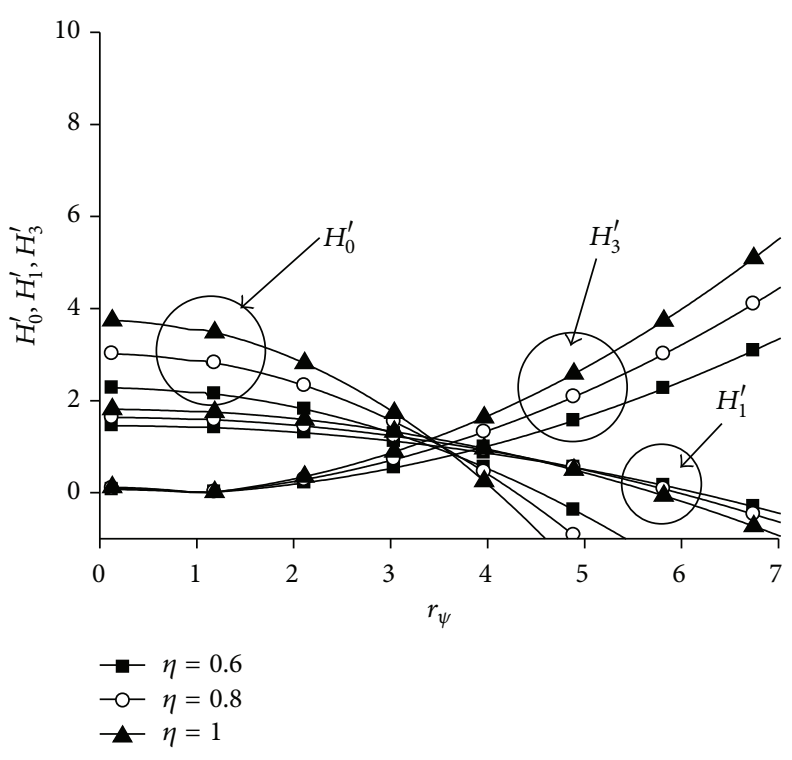

(b)

FIgURE 7: Coefficients of stability: (a) $r_{m}=0.03$; (b) $r_{m}=0.09$.

From (21), $r_{\psi}-2 \alpha$ plane is divided into two regions of $180^{\circ}$ and $0^{\circ} .2 \alpha$ changes at $r_{\psi}=1$ with every $\eta$. If the structure parameters of the vibration system are completely symmetrical, such as the mass-radius product of two ERs, $2 \alpha$ achieves the change of $180^{\circ}$ to $0^{\circ}$ with increasing $r_{\psi}$. By comparing $2 \alpha$ with $\eta$, the better the symmetry of the structural parameter of the vibration system is, the easier $2 \alpha$ approach $180^{\circ}$ and $0^{\circ}$.

For further study of the effect of the structural parameters of the vibration system on the synchronization stability, we apply bisection method to obtain the stability domains of the synchronized motion. Figure 7 shows the changes of the coefficients of the synchronization stability $H_{0}^{\prime}, H_{1}^{\prime}$, and $H_{3}^{\prime}$ with the different mass ratio $\eta$ between ERs. From the stability condition, we know that the vibration system runs in the steady state when $H_{0}^{\prime}>0, H_{1}^{\prime}>0$, and $H_{3}^{\prime}>0$. Since $H_{3}^{\prime}$ is the function of $W_{c}$, it has zero value at $r_{\psi}=1$. By comparing the changes of $H_{0}^{\prime}, H_{1}^{\prime}$, and $H_{3}^{\prime}$, it can be seen that $H_{3}^{\prime}$ is always greater than zero. In the region of the phase difference tending to $0^{\circ}, H_{3}^{\prime}$ increases with increasing $r_{\psi}$, and $H_{0}^{\prime}$ and $H_{1}^{\prime}$ decrease with increasing $r_{\psi}$. It is noteworthy that $H_{0}^{\prime}$ goes earlier through zero than $H_{1}^{\prime}$. According to the stability condition, the vibration system is unstable at the moment. Comparing comprehensively $H_{0}^{\prime}, H_{1}^{\prime}$, and $H_{3}^{\prime}$, we know that the vibration system can operate in steady state when $H_{0}^{\prime}>0$. Therefore, the stability condition of two ERs achieving the synchronized motion is $H_{0}^{\prime}>0$. In addition, the smaller $r_{m}$, the bigger the synchronization stability of the vibration system.

\section{Experiments}

In this section, we will verify the validity of the dynamic model, theoretical analysis, and numerical results of the above sections, by comparing to experimental results for

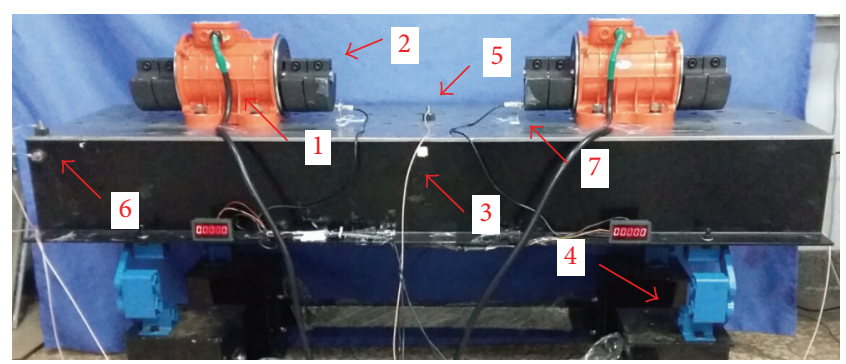

FIGURE 8: Experiment equipment $\left(r_{\psi} \approx 0.6\right)$.

a laboratory model. Figure 8 shows the mechanical composition of the laboratory model, which consists of two AC vibration motors, the rigid vibration base, the springs, and the support base. Number 1 represents AC motor, number 2 represents ER, number 3 represents the rigid vibration base, number 4 represents the rubber spring, number 5 and number 6 represent the positions measured by the acceleration sensors, and number 7 represents the angular velocity measured by Hall sensors. The angular velocities of two motors and the signals of the vibrations in the vertical and horizontal directions are collected by NI data acquisition card, while two motors operate in $45 \mathrm{~Hz}$ power frequency by Siemens inverters.

As shown in Figure 9, $r_{\psi} \approx 0.6$, motor 1 first runs in the starting process, and motor 2 begins to run after $3 \mathrm{~s}$. Because of the identical motors and the same inertial moments of two ERs $(\eta=1)$, the synchronization torque begins to adjust the phase difference between two ERs to achieve the synchronized motion, with approaching the rotational velocities of two motors. Since the adjustment of the synchronization torque, the synchronization velocity and the phase difference 


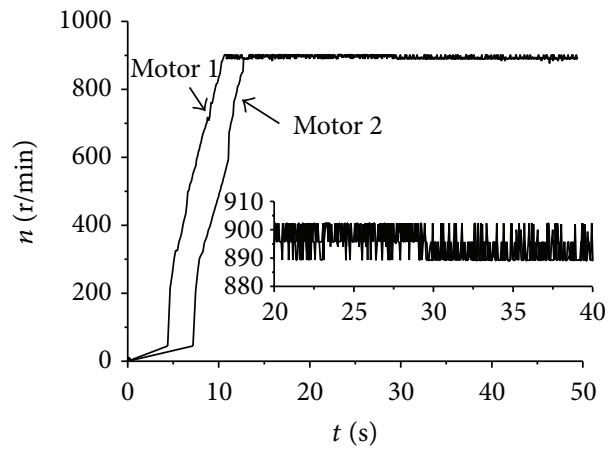

(a)

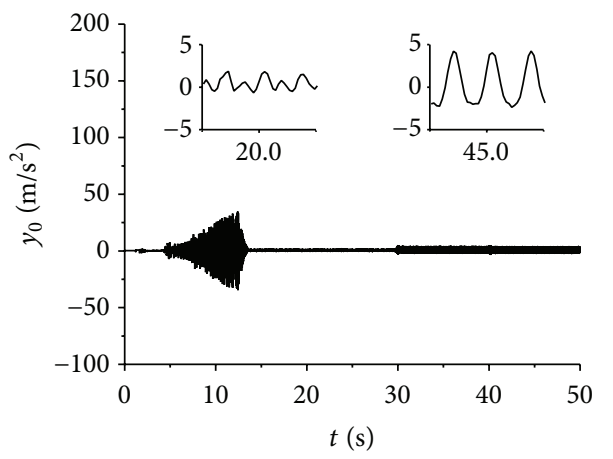

(c)

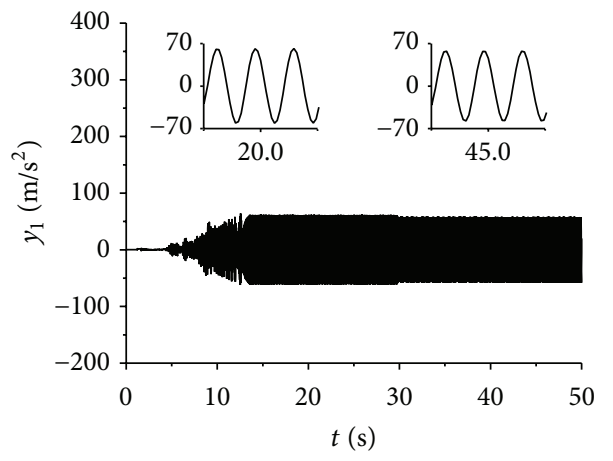

(e)

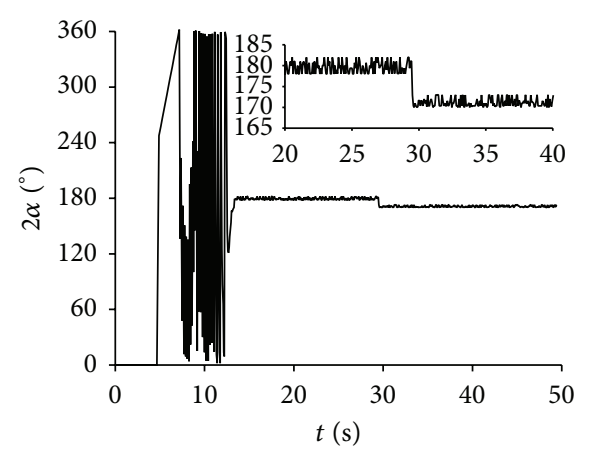

(b)

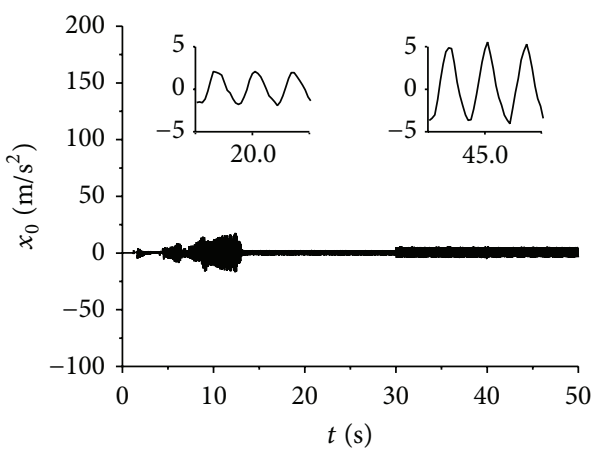

(d)

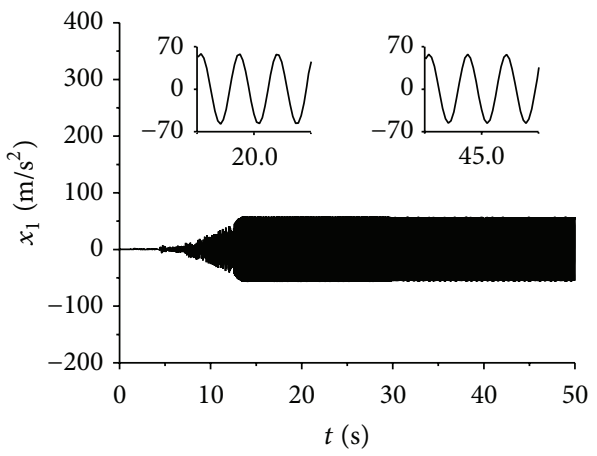

(f)

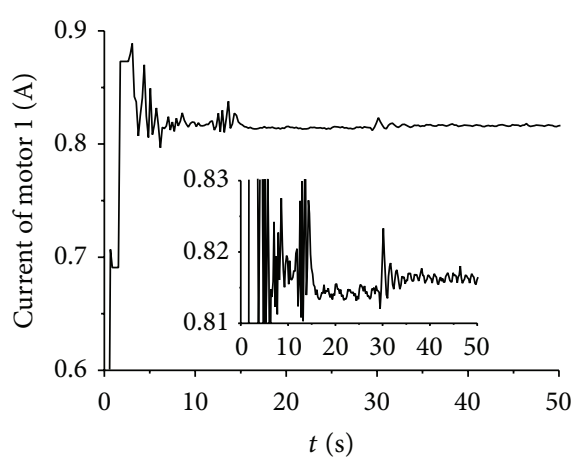

(g)

FIGURE 9: Experiment results with $r_{\psi} \approx 0.6, \eta=1$, and $r_{m} \approx 0.03$ : (a) rotational velocities; (b) the phase difference; (c) response in $y$-direction for number 5; (d) response in $x$-direction for number 5; (e) response in $y$-direction for number 6; (f) response in $x$-direction for number 6; (g) current of motor 1. 
are stabilizing fast, as shown in Figures 9(a) and 9(b), the synchronization velocity is about $n \approx 896 \mathrm{r} / \mathrm{min}$, and the phase difference is about $2 \alpha \approx 180^{\circ}$. Due to the fact that two ERs operate in far-resonant, they excite the resonant responses through the resonant region of the vibration system in $x$ - and $y$-directions, as shown in Figures 9(c)-9(f). Figures 9(c) and 9(d) show the responses of the position for number 5, and Figures 9(e) and 9(f) show the responses of the position for number 6 . From comparison between the response of number 5 and that of number 6 , it can be seen that the main motion type of the vibration system is the spatial cone motion.

At about $30 \mathrm{~s}$, the power of motor 2 is switched off, which breaks the original synchronization state between two ERs. Under the function of the synchronization torque, the vibration system can achieve another synchronization state by adjusting the phase difference. This phenomenon is called the vibration synchronization transmission [1]. Figure 9 shows that, after switching off the power of motor 2 , the synchronization velocity is about $n \approx 892 \mathrm{r} / \mathrm{min}$, and the phase difference is about $2 \alpha \approx 172^{\circ}$. The responses of number 5 are slightly larger, and those of number 6 are slightly smaller.

Figure $9(\mathrm{~g})$ shows the root mean square of stator current of motor 1 . Since the load torque of motor 1 is the biggest at rest, there is a peak value on the current curve at the beginning of power supply, and the current begins to decrease after motor 1 rotating. There is another peak value of current before the moment when synchronized motion is carried out, and the current curve is fluctuating because the phase of motor 2 lags behind that of motor 1 . The synchronization torque acts the load torque on motor 1 , which increases the current of motor 1. After the moment synchronized motion is achieved, the current curve becomes smooth, which illustrates that the vibration system operates in the steady synchronization state because the load torque of motor 1 is close to constant. At about $30 \mathrm{~s}$, the power of motor 2 is switched off. The current value of motor 1 increases with increasing phase difference, but the current value is still smooth under the synchronized motion. According to the change of current, we know the energy distribution of the vibration system. In other words, we can see how the power consumption changes after cutting off motor 2 by observing the change of current of motor 1 .

As shown in Figure 10, $r_{\psi} \approx 1.1$, motor 1 first runs in the starting process, and motor 2 begins to run after $3 \mathrm{~s}$. Figures 10 (a) and 10(b) show that the synchronization velocity is about $n \approx 896 \mathrm{r} / \mathrm{min}$, and the phase difference is about $2 \alpha \approx 0^{\circ}$. In the starting process, the phase difference oscillates between $0^{\circ}$ and $180^{\circ}$ because of the difference in rotational velocities of two motors. Similar to scheme in Figures 9(c)9(f), Figure 10(c) and Figure 10(d) show the responses of the position for number 5, and Figure 10(e) and Figure 10(f) show the responses of the position for number 6 . From comparison of the response of number 5 and number 6 , it can be seen that the main motion type of the vibration system is the spatial circle motion. Due to the different type of the vibration system, the responses of number 6 in Figure 10 are greater than that in Figure 9.

At about $27 \mathrm{~s}$, the power of motor 2 is switched off, which breaks the original synchronization state between two
ERs. Since the condition of the vibration synchronization transmission cannot be satisfied, the rotational velocity of motor 2 rapidly decreases, oscillation occurs in the phase difference, and the responses of number 5 and number 6 also show irregularity oscillation, as shown in Figure 9. After $3 \mathrm{~s}$, the power of motor 2 is switched on. The vibration system again implements the synchronized motion. The new synchronization state is same with the old synchronization state.

From the above two groups of experiments, we know that the initial state of two motors has no effect on the synchronization state. When the synchronization torque is large enough, the vibration synchronization transmission can be achieved. As shown in Figure 4, the synchronization torque of $r_{\psi} \approx 0.6$ is greater than that of $r_{\psi} \approx 1.1$. So the vibration system with $r_{\psi} \approx 0.6$ can achieve the vibration synchronization transmission and that with $r_{\psi} \approx 1.1$ cannot. When $r_{\psi} \approx 0.6$, the motion type of the vibration system is the spatial cone motion, while when $r_{\psi} \approx 1.1$, the motion type of the vibration system is the spatial circle motion, which comply with Figure 6. From Figure 7, we know that when $r_{\psi} \ll 4$, the synchronized motion of the vibration system is stable, which conforms to the responses of number 5 and number 6 . The comparison between numeric results and experiments shows they are very good consistency. Based on experiments, the validity of theoretical analysis is proved, which helps the design of this type of vibration machine.

\section{Conclusions}

Based on the theoretical analysis and experiment result, the following remarks should be explained.

With introducing the average method of small parameters, synchronization of two ERs with the common rotational axis in the vibration system of the far-resonant spatial motion is investigated. Based on the dimensionless coupling equation, the synchronization condition of two ERs is obtained, and the stability condition of two ERs achieving the synchronized motion is derived, which satisfies RouthHurwitz criterion. The two conditions can be used to evaluate and discriminate whether the vibration system is able to carry out the synchronized motion.

The coupling dynamic characteristic, such as the choosing motion feature of the vibration system, is discussed by numerical method. Synchronization of two ERs depends on the coupling dynamic characteristic. The better the symmetry for two ERs, the greater the synchronization torque, the easier the achievement of the synchronized motion, and the stronger the synchronization ability. In addition, $r_{m}$ should be as small as possible for strengthening the stability of the synchronized motion.

From experiments, it can be seen that if $r_{\psi}<1$, the spatial cone motion of the vibration system forces the phase difference to approach $\pi$, and if $r_{\psi}>1$, the spatial circle motion of the vibration system forces the phase difference to approach zero. In engineering, $l_{0}$ should be selected as far as possible. Only in this way we can obtain the circle motion of spatial for this type of vibration machine. 


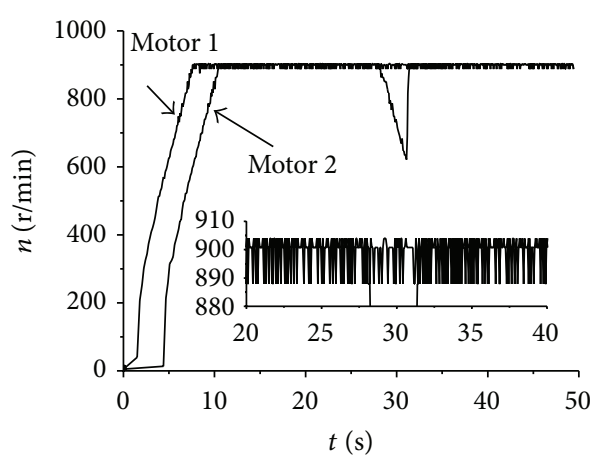

(a)

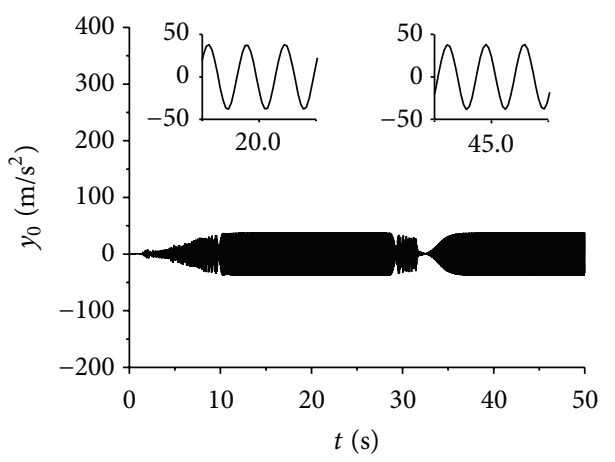

(c)

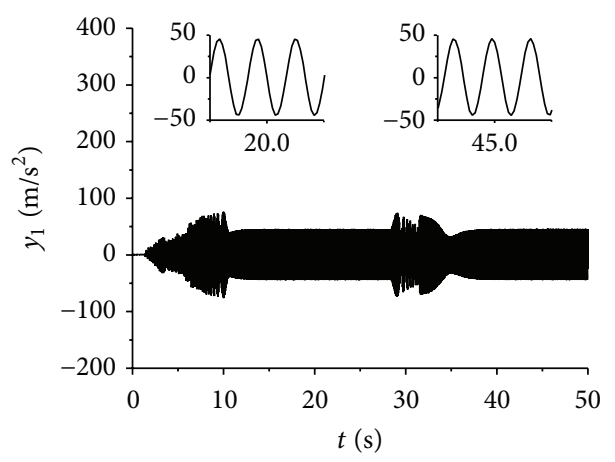

(e)

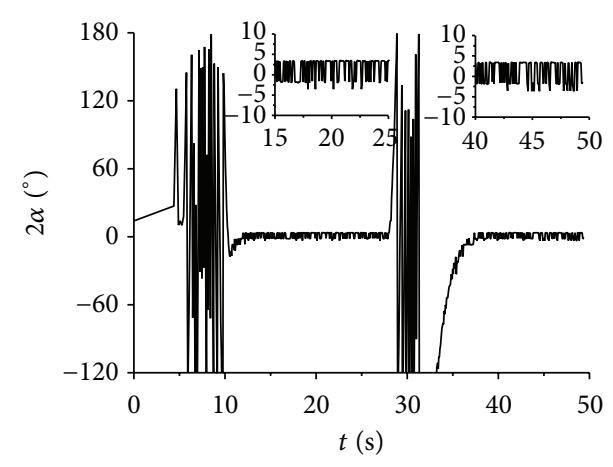

(b)

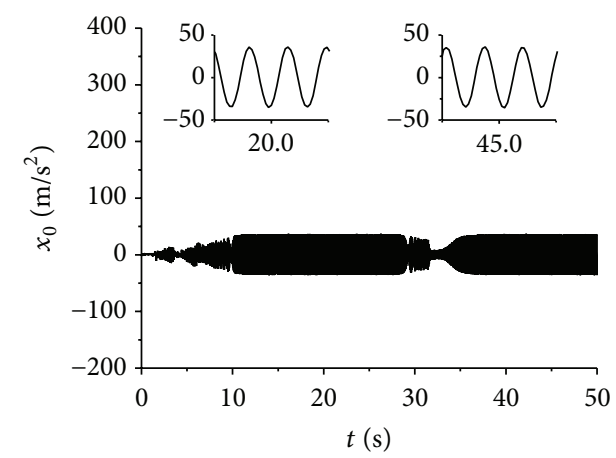

(d)

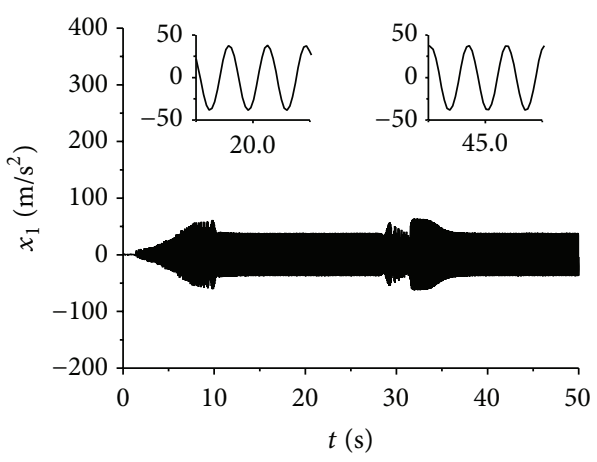

(f)

FIGURE 10: Experiment results with $r_{\psi} \approx 1.1, \eta=1$, and $r_{m} \approx 0.03$ : (a) rotational velocities; (b) the phase difference; (c) response in $y$-direction for number 5; (d) response in $x$-direction for number 5; (e) response in $y$-direction for number 6 ; (f) response in $x$-direction for number 6.

Comparing theoretical analysis, numerical results, and experiments, the feasibility of the theory method in this paper is proved.

\section{Appendix}

\section{A. Rotation Matrix}

According to Cartesian coordinates, the rotation matrix is [19]

$$
\mathbf{R}_{1}^{\mathrm{T}}=\left[\begin{array}{ccc}
\cos \theta & 0 & -\sin \theta \\
\sin \theta \sin \psi & \cos \psi & \cos \theta \sin \psi \\
\cos \psi \sin \theta & -\sin \psi & \cos \theta \cos \psi
\end{array}\right]^{\mathrm{T}}
$$

Under the hypothesis of small angel $\psi$ and $\theta$, the matrix can be simplified as

$$
\mathbf{R}_{2}^{\mathrm{T}}=\left[\begin{array}{ccc}
1 & 0 & -\theta \\
0 & 1 & \psi \\
\theta & -\psi & 1
\end{array}\right]^{\mathrm{T}}
$$

\section{B. Dimensionless Parameters}

Consider the following:

$$
\begin{aligned}
& \chi_{f 1}=\frac{1}{2} m_{0} r^{2} \omega_{m 0}^{2}\left(W_{s 0}+W_{s} \cos 2 \alpha\right), \\
& \chi_{f 2}=\frac{1}{2} m_{0} r^{2} \omega_{m 0}^{2}\left(\eta W_{s 0}+W_{s} \cos 2 \alpha\right),
\end{aligned}
$$




$$
\begin{aligned}
& \chi_{a}=\frac{1}{2} m_{0} r^{2} \omega_{m 0}^{2} W_{c} \sin 2 \alpha, \\
& \chi_{11}^{\prime}=\frac{1}{2} m_{0} r^{2} \omega_{m 0}\left(W_{c 0}-W_{s} \sin 2 \alpha+W_{c} \cos 2 \alpha\right), \\
& \chi_{12}^{\prime}=\frac{1}{2} m_{0} r^{2} \omega_{m 0}\left(W_{c 0}+W_{s} \sin 2 \alpha-W_{c} \cos 2 \alpha\right), \\
& \chi_{21}^{\prime}=\frac{1}{2} m_{0} r^{2} \omega_{m 0}\left(\eta^{2} W_{c 0}+W_{s} \sin 2 \alpha+W_{c} \cos 2 \alpha\right), \\
& \chi_{22}^{\prime}=\frac{1}{2} m_{0} r^{2} \omega_{m 0}\left(-\eta^{2} W_{c 0}+W_{s} \sin 2 \alpha+W_{c} \cos 2 \alpha\right), \\
& \chi_{11}=m_{0} r^{2} \omega_{m 0}^{2}\left(W_{s 0}+W_{s} \cos 2 \alpha+W_{c} \sin 2 \alpha\right), \\
& \chi_{12}=m_{0} r^{2} \omega_{m 0}^{2}\left(W_{s 0}-W_{s} \cos 2 \alpha-W_{c} \sin 2 \alpha\right), \\
& \chi_{21}=m_{0} r^{2} \omega_{m 0}^{2}\left(\eta^{2} W_{s 0}+W_{s} \cos 2 \alpha-W_{c} \sin 2 \alpha\right), \\
& \chi_{22}=m_{0} r^{2} \omega_{m 0}^{2}\left(-\eta^{2} W_{s 0}+W_{s} \cos 2 \alpha-W_{c} \sin 2 \alpha\right), \\
& W_{c 0} \\
& =-r_{m}\left(\frac{\cos \gamma_{x}}{\mu_{x}}+\frac{\cos \gamma_{y}}{\mu_{y}}+r_{\psi}^{2} \frac{\cos \gamma_{\psi}}{\mu_{\psi}}+r_{\theta}^{2} \frac{\cos \gamma_{\theta}}{\mu_{\theta}}\right),
\end{aligned}
$$$$
W_{c}
$$$$
=-r_{m} \eta\left(\frac{\cos \gamma_{x}}{\mu_{x}}+\frac{\cos \gamma_{y}}{\mu_{y}}-r_{\psi}^{2} \frac{\cos \gamma_{\psi}}{\mu_{\psi}}-r_{\theta}^{2} \frac{\cos \gamma_{\theta}}{\mu_{\theta}}\right),
$$$$
W_{s 0}=r_{m}\left(\frac{\sin \gamma_{x}}{\mu_{x}}+\frac{\sin \gamma_{y}}{\mu_{y}}+r_{\psi}^{2} \frac{\sin \gamma_{\psi}}{\mu_{\psi}}+r_{\psi}^{2} \frac{\sin \gamma_{\theta}}{\mu_{\theta}}\right) \text {, }
$$$$
W_{s}=r_{m} \eta\left(\frac{\sin \gamma_{x}}{\mu_{x}}+\frac{\sin \gamma_{y}}{\mu_{y}}-r_{\psi}^{2} \frac{\sin \gamma_{\psi}}{\mu_{\psi}}-r_{\theta}^{2} \frac{\sin \gamma_{\theta}}{\mu_{\theta}}\right) \text {. }
$$

\section{Competing Interests}

The authors declare that there are no competing interests regarding the publication of this paper.

\section{Acknowledgments}

This work is supported by National Natural Science Foundation of China, Grants nos. 51305070 and 51375080.

\section{References}

[1] B. C. Wen, J. Fan, C. Y. Zhao, and W. L. Xiong, Vibratory Synchronization and Controlled Synchronization in Engineering, Science Press, Beijing, China, 2009.

[2] C. Huygens, Horologium Oscillatorium, F. Muguet, Paris, France, 1673.

[3] I. I. Blekhman, "Self-synchronization of vibrators in some types of vibrational machines," Inzhenerny Sbornik, vol. 16, pp. 49-72, 1953 (Russian).
[4] I. I. Blekhman, A. L. Fradkov, H. Nijmeijer, and A. Y. Pogromsky, "On self-synchronization and controlled synchronization," Systems \& Control Letters, vol. 31, no. 5, pp. 299-305, 1997.

[5] I. I. Blekhman, Synchronization in Science and Technology, ASME Press, New York, NY, USA, 1988.

[6] I. I. Blekhman, A. L. Fradkov, O. P. Tomchina, and D. E. Bogdanov, "Self-synchronization and controlled synchronization: general definition and example design," Mathematics and Computers in Simulation, vol. 58, no. 4-6, pp. 367-384, 2002.

[7] I. I. Blekhman and N. P. Yaroshevich, "Extension of the domain of applicability of the integral stability criterion (extremum property) in synchronization problems," Journal of Applied Mathematics and Mechanics, vol. 68, no. 6, pp. 839-846, 2004.

[8] I. I. Blekhman and V. S. Sorokin, "On the separation of fast and slow motions in mechanical systems with high-frequency modulation of the dissipation coefficient," Journal of Sound and Vibration, vol. 329, no. 23, pp. 4936-4949, 2010.

[9] C. Y. Zhao, H. T. Zhu, R. Z. Wang, and B. C. Wen, "Synchronization of two non-identical coupled exciters in a non-resonant vibrating system of linear motion. Part I: theoretical analysis," Shock and Vibration, vol. 16, no. 5, pp. 505-515, 2009.

[10] C. Y. Zhao, H. T. Zhu, T. J. Bai, and B. C. Wen, "Synchronization of two non-identical coupled exciters in a non-resonant vibrating system of linear motion. Part II: numeric analysis," Shock and Vibration, vol. 16, no. 5, pp. 517-528, 2009.

[11] C. Y. Zhao, Q. H. Zhao, Y. M. Zhang, and B. C. Wen, "Synchronization of two non-identical coupled exciters in a non-resonant vibrating system of plane motion," Journal of Mechanical Science and Technology, vol. 25, no. 1, pp. 49-60, 2011.

[12] X.-L. Zhang, C.-Y. Zhao, and B.-C. Wen, "Theoretical and experimental study on synchronization of the two homodromy exciters in a non-resonant vibrating system," Shock and Vibration, vol. 20, no. 2, pp. 327-340, 2013.

[13] X. L. Zhang, B. C. Wen, and C. Y. Zhao, "Vibratory synchronization transmission of two exciters in a super-resonant vibrating system," Journal of Mechanical Science and Technology, vol. 28, no. 6, pp. 2049-2058, 2014.

[14] X. L. Zhang, B. C. Wen, and C. Y. Zhao, "Synchronization of three non-identical coupled exciters with the same rotating directions in a far-resonant vibrating system," Journal of Sound and Vibration, vol. 332, no. 9, pp. 2300-2317, 2013.

[15] J. M. Balthazar, J. L. P. Felix, and R. M. L. R. F. Brasil, "Some comments on the numerical simulation of self-synchronization of four non-ideal exciters," Applied Mathematics and Computation, vol. 164, no. 2, pp. 615-625, 2005.

[16] J. M. Balthazar, J. L. P. Felix, and R. M. L. R. F. Brasil, "Short comments on self-synchronization of two non-ideal sources supported by a flexible portal frame structure," JVC/Journal of Vibration and Control, vol. 10, no. 12, pp. 1739-1748, 2004.

[17] Y. Bonkobara, T. Ono, and T. Kondou, "Development of a generation mechanism of synchronous vibration suitable for hand-held vibrating tools: investigation of an impact model with two oscillators," Journal of System Design and Dynamics, vol. 5, no. 6, pp. 1361-1375, 2011.

[18] S. Rumyantsev and D. Tarasov, "Numerical simulation of nonlinear dynamics of vibration transport machines in case of three independently rotating vibration exciters: recent advances in applied mathematics," in Proceedings of the American Conference on Applied Mathematics (AMERICAN-MATH '10), pp. 191194, Cambridge, Mass, USA, January 2010. 
[19] C. Y. Zhao, H. T. Zhu, Y. M. Zhang, and B. C. Wen, "Synchronization of two coupled exciters in a vibrating system of spatial motion," Acta Mechanica Sinica, vol. 26, no. 3, pp. 477-493, 2010.

[20] Á. Miklós and Z. Szabó, "Vibrator with DC motor driven eccentric rotors," Periodica Polytechnica, Mechanical Engineering, vol. 56, no. 1, pp. 49-53, 2012.

[21] Á. Miklós and Z. Szabó, "Mechanical synchronization in dualrotor vibroactuator," Proceedings in Applied Mathematics and Mechanics, vol. 13, no. 1, pp. 41-42, 2013.

[22] A. Miklós and Z. Szabó, "Simulation and experimental validation of the dynamical model of a dual-rotor vibrotactor," Journal of Sound and Vibration, vol. 334, pp. 98-107, 2015.

[23] Y. Ruan and B. S. Chen, Control Systems of Electric DerivesMotion Control Systems, China Machine Press, Beijing, China, 2010 (Chinese). 


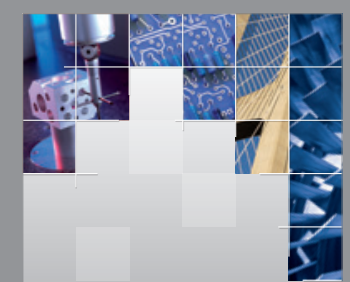

\section{Enfincering}
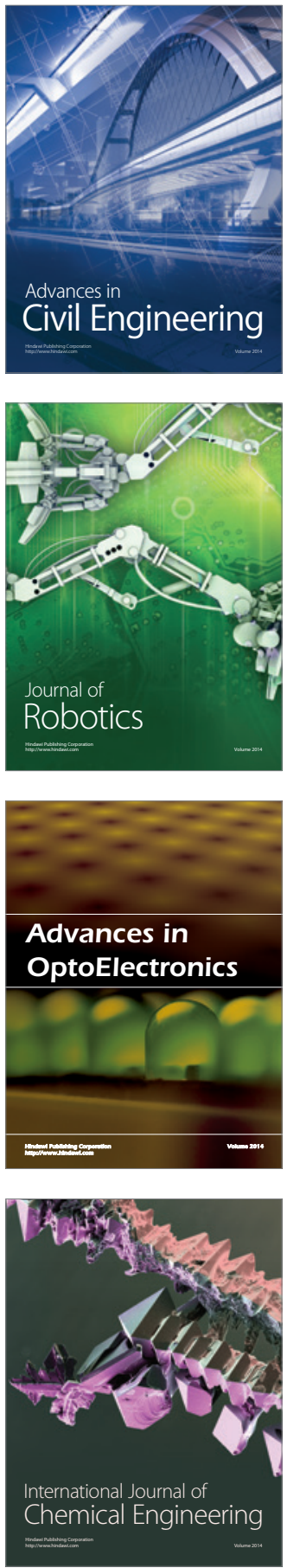

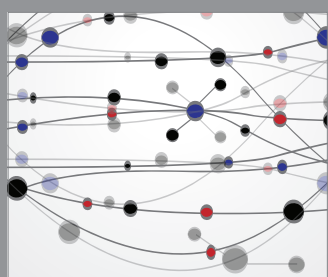

The Scientific World Journal

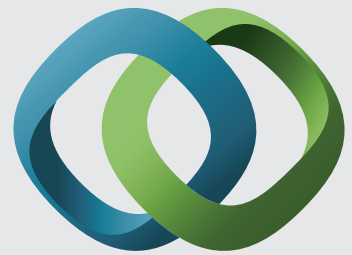

\section{Hindawi}

Submit your manuscripts at

http://www.hindawi.com
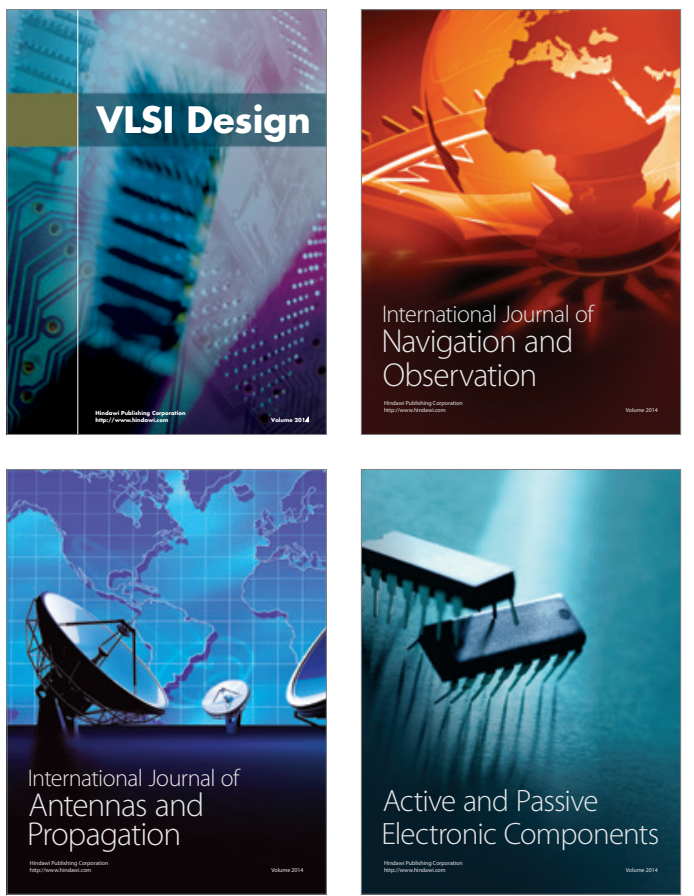
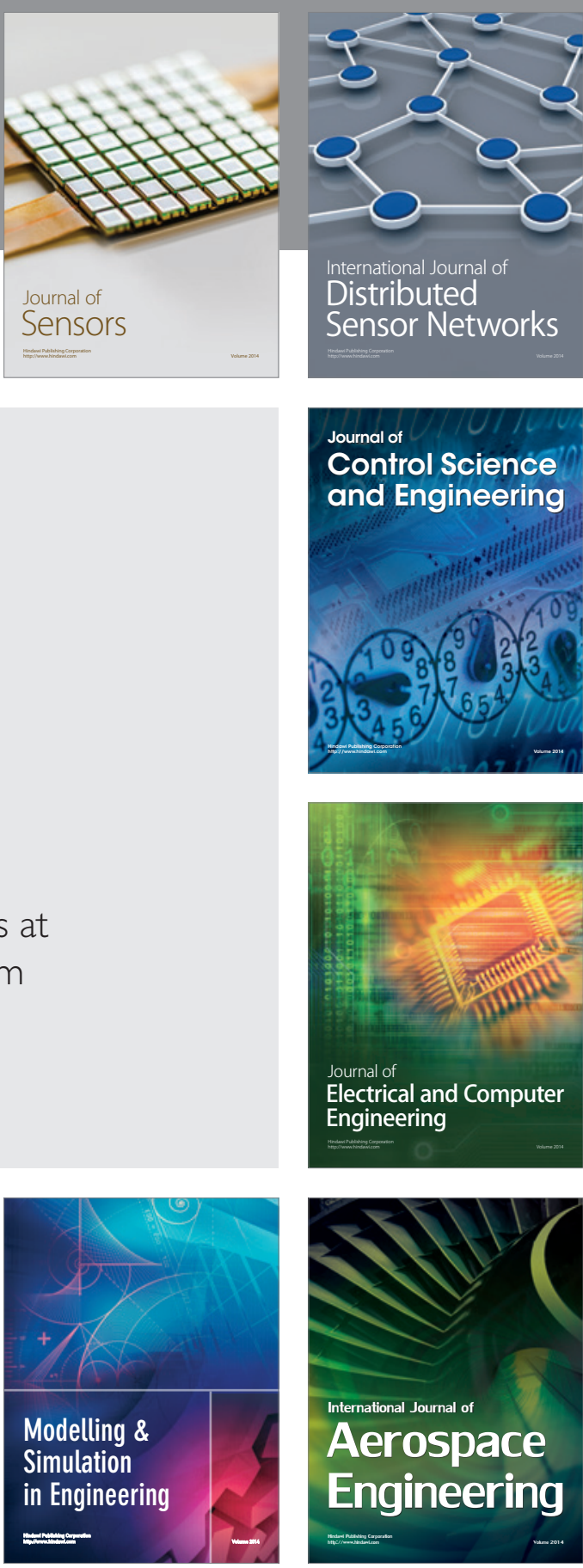

International Journal of

Distributed

Sensor Networks

Journal of

Control Science

and Engineering
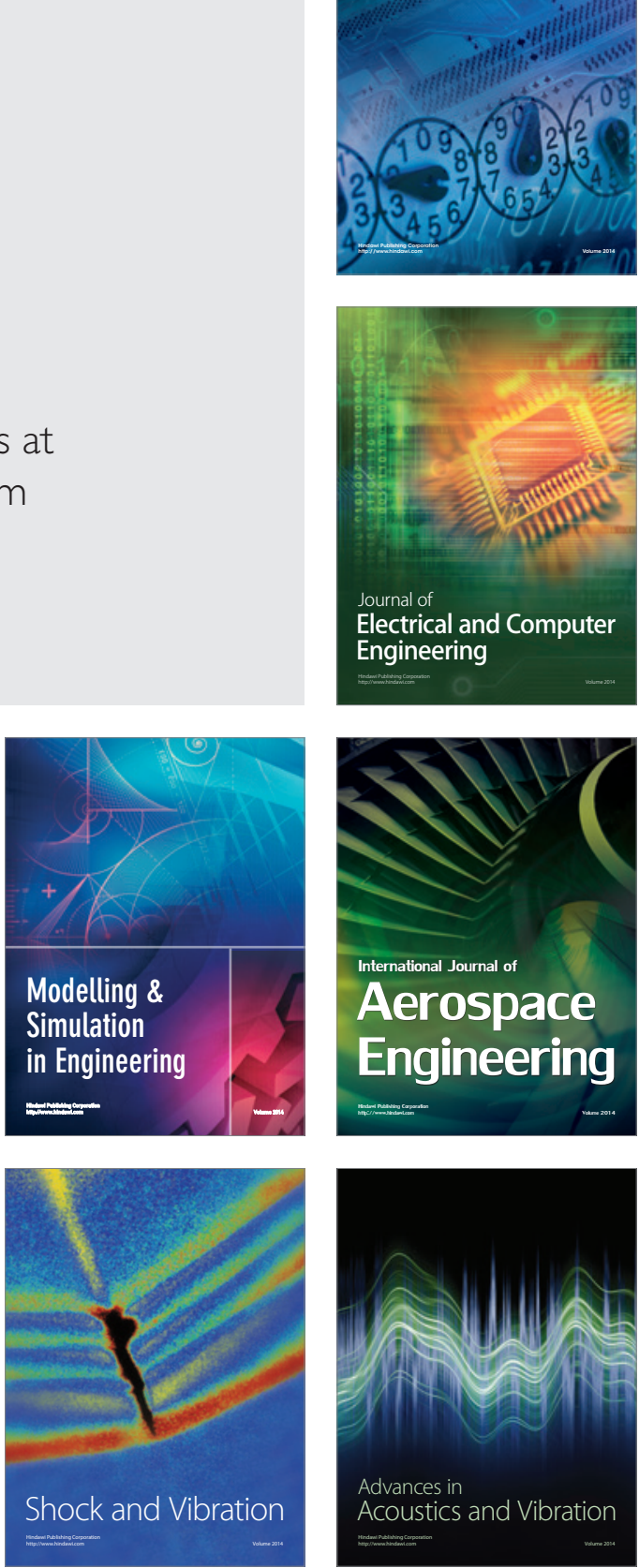